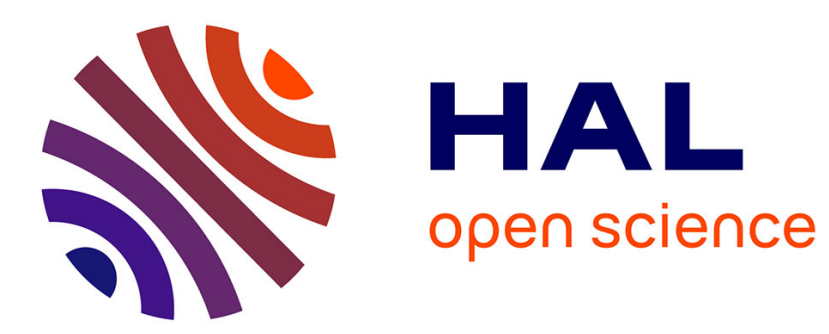

\title{
Sensing and computational frameworks for improving drill-string dynamics estimation
}

Jean Auriol, Nasser Kazemi, Silviu-Iulian Niculescu

\section{To cite this version:}

Jean Auriol, Nasser Kazemi, Silviu-Iulian Niculescu. Sensing and computational frameworks for improving drill-string dynamics estimation. Mechanical Systems and Signal Processing, 2021, 10.1016/j.ymssp.2021.107836 . hal-03167464

\section{HAL Id: hal-03167464 \\ https://hal.science/hal-03167464}

Submitted on 12 Mar 2021

HAL is a multi-disciplinary open access archive for the deposit and dissemination of scientific research documents, whether they are published or not. The documents may come from teaching and research institutions in France or abroad, or from public or private research centers.
L'archive ouverte pluridisciplinaire HAL, est destinée au dépôt et à la diffusion de documents scientifiques de niveau recherche, publiés ou non, émanant des établissements d'enseignement et de recherche français ou étrangers, des laboratoires publics ou privés. 


\title{
Sensing and computational frameworks for improving drill-string dynamics estimation
}

\author{
Jean Auriol ${ }^{*, a}$, Nasser Kazemi ${ }^{\mathrm{b}}$, Silviu-Iulian Niculescu ${ }^{\mathrm{a}}$ \\ ${ }^{a}$ Université Paris-Saclay, CNRS, CentraleSupélec, Laboratoire des signaux et systèmes, Gif-sur-Yvette, France. \\ ${ }^{b}$ Department of Chemical and Petroleum Engineering, University of Calgary, Canada.
}

\begin{abstract}
In this paper, we consider the axial motion of a directional multi-sectional drill-string. The drillstring dynamics are represented by a distributed dynamical model (wave equations) coupled with an ordinary differential equation at the downhole boundary (bit-rock interaction). The interaction between the drill-bit and rock can introduce severe vibrations in the drill-string and result in safety and performance issues. Consequently, the performance of drilling is interwoven with our knowledge of the subsurface. To address these problems, we propose a sensing and computational framework for estimating the drill-string dynamics and the specific intrinsic energy of the rocks while drilling. By exploiting the derived models' particular structure, we combine the drill-string dynamics modeling with top-drive hook-load (force) and hook-speed (velocity) measurements to estimate the force-on-bit without requiring the knowledge of the sub-surface. Then, we record and model the seismic radiation patterns of drill-bit rock interactions near the surface (i.e., seismic while drilling). Such an idea allows deriving an appropriate estimation of the rocks' intrinsic energy while drilling. We introduce two alternative rock property estimation algorithms based on direct parameter estimation and machine learning concepts to complete the analysis. The different approaches are tested and validated in simulations. We discuss their respective advantages and drawbacks. Finally, we show how to extend our methodologies in the presence of non-linear Coulomb friction terms and of coupled axial-torsional oscillations.
\end{abstract}

Key words: Dynamical system, Distributed system, backstepping, Seismic-While-Drilling, Parameter estimation, Neural Networks

\section{Introduction}

Knowledge of the subsurface reservoirs, such as hydrocarbons, minerals, and geothermal, and an efficient method for accessing them are crucial in exploring and exploiting the reserves. Accessing the reservoirs involves a drilling process. In drilling, the interaction with the rock can generate 5 significant vibrations and result in an inefficient Rate Of Penetration (ROP). It may also raise safety issues. Control mechanisms may reduce these vibrations in the system [? ? ]. However, such control mechanisms often depend on the properties of the drilled rock. In this paper, we design three algorithms that provide a near-real-time estimation of the rock's nature for a directional multi-sectional well.

\footnotetext{
${ }^{*}$ Corresponding author

Email address: jean.auriol@12s.centralesupelec.fr (Jean Auriol)

Preprint submitted to Mechanical Systems and Signal Processing
}

March 12, 2021 


\subsection{Insights in modeling drill-string dynamics: Complexity and related issues}

Drill-string dynamic behavior often manifests itself as drill string vibrations. These vibrations have been extensively studied in the literature [? ? ? ? ]. Among them, torsional oscillations, known as stick-slip, are characterized by a series of stopping - "sticking" - and releasing - "slipping" - events of the bit. These oscillations can reduce the Rate of Penetration, damage the well by causing fatigue on the equipment, and eventually lead to premature failure of the bit [? ]. Such oscillations are caused by Coulomb friction-induced side forces [? ] and by non-linear frictional force actuating at the bit by contact with the rock [? ? ]. Indeed, numerous models consider those stick-slip oscillations are related to the velocity-weakening of the frictional force at the bit (Stribecklike effect) associated with typical dry friction profiles (static friction and dynamic friction) [? ? ]. However, the stick-slip phenomenon can also occur off-bottom and does not require a velocity weakening in the bit-rock interaction [? ? ? ]. In this context, to reduce the harmful effects of such vibrations and improve the drilling device's performance, it appears necessary to clearly understand the dynamics of the drill-string. In [? ? ], the axial and torsional dynamics are described by a set of hyperbolic Partial Differential Equations (PDEs). These equations are usually governed by wave equations combined with gravitational terms and a non-linear Coulomb side-force [? ]. In the case of multi-sectional wells, the change of the characteristic line impedance between each section may reflect the traveling waves [? ? ]. These reflections are taken into account in the model using interconnection between the PDEs [? ]. Such a class of distributed models has been extensively analyzed in [? ? ]. A validation against field data for the corresponding torsional model has been proposed in [? ]. Regarding the boundary conditions, specific attention must be paid to the bit-rock interaction law at the downhole boundary [? ? ? ]. Different models have been proposed in the literature [? ] to represent the contact between the cutting device and the drilling surface. For instance, the approach developed in [? ] for drag bits and adjusted in [? ] for roller-cone bit divides the tool's action into three independent processes: a pure cutting process, a pure indentation process, and a frictional contact process. Such a model is shown to be consistent with laboratory results in [? ]. All in all, the drilling system can be modeled as interconnected hyperbolic PDEs coupled with Ordinary Differential Equations (ODEs) at their boundaries. In that sense, they are related to the larger class of flexible systems for which extensive literature already exist [? ? ? ? ]. Note that other models have been proposed in [? ? ] for percussive drilling systems.or When trying to deal with vibration control for drilling systems, a problem that naturally arises is that such drilling models depend on the nature of the drilled rock (which is a priori unknown) The dependence of the drill-string dynamic response to the bit-rock interaction and rock properties is verified in [? ]. Accordingly, estimating the drilled rock's characteristics (such as its seismic velocity or its intrinsic specific energy) appears necessary to improve drilling devices' performance. Estimating rocks' seismic velocities while drilling is not an easy task as downhole sensors are expensive and may raise potential technical risks. Thus, the disturbance observer developed in [? ? ? ] cannot be straightforwardly adjusted (since the downhole measurements are not available). To address this problem, an approach based on seismic while drilling (SWD) has been proposed in [? ]. Seismic-while-drilling is a technique that records the seismic energy of the drill-bit rock interactions in the nearby well locations or near the surface [? ]. SWD has several applications. For example, SWD measurements can provide the drill-bit position through check shots or sonic calibrations through reflectivity characterization [? ]. Imaging SWD data can provide subsurface structure around and ahead of the drill-bit [? ? ]. Moreover, full-waveform inversion of SWD data can result in the high-resolution estimation of rocks' seismic properties in 
the sub-surface [? ]. Here, we extend the formation seismic velocity estimation method of [? ], which is designed for uni-sectional vertical well, to a directional multi-sectional well without considering Coulomb friction or torsional motion. In drilling environments where recording the SWD data is not feasible or lacks quality, we need to explore alternative options. Since the method developed in [? ] provides an expression of the downhole force and velocity as functions of topdrive measurements, it is possible to use classical parameter estimations techniques [? ] on the downhole boundary condition to estimate the rock properties. Also, machine learning approaches are efficient in estimating parameters based on available measurement signals [? ? ].

\subsection{Towards a unified methodology of modeling, sensing, analysis, and control}

One of the practical problems arising in drilling consists of deriving an appropriate real-time estimation of the nature of the drilled rock. To the best of the authors' knowledge, such a problem has not received a satisfactory answer in the open literature. In our opinion, the proposed results and underlying ideas represent a new step in deriving an appropriate engineering-based solution. More precisely, the main contributions of the paper are twofold: (i) first, improving the drill-string dynamics parameters' estimations of the nature of the drilled rock in some unified dynamical model based on a better understanding of the underlying transport phenomena by exploiting the dynamics interconnection as well as the interconnection structure of the corresponding process; (ii) second, explicitly presenting three algorithms to estimate the seismic velocity of the rock, the energy of the rock interacting with the drill-bit.

To improve the parameter estimation, we will propose a recursive dynamics interconnection framework allowing us to reduce the existing gap between modeling and estimation in the context of complex dynamics as whose described by PDEs to represent drilling dynamics. In our opinion, such a recursive interconnection framework is better adapted to sensing and estimation since it allows structuring the model on some simpler and more realistic interconnected dynamical subsystems (blocks) and better exploiting the structure of the interconnection. To the best of the authors' nowledge, this framework represents a novelty in the open literature allowing a global estimation, analysis, and control of the corresponding process with a reduced computational cost. It is worth mentioning that the computational effort is essential when one of the focuses is on real-time estimations. Finally, this framework is a natural environment to develop estimation techniques (as it is done in this paper) or to solve control problems (for instance by adjusting the recent Lyapunov techniques developed for flexible systems in [? ? ? ? ] or the thrust force control methodology developed in [?]), to develop efficient state-observers [? ].

Second, in the proposed framework, we present three algorithms to estimate the specific intrinsic energy of the rock interacting with the drill-bit. We consider the case of the axial motion of a directional multi-sectional well, for which we neglect the Coulomb friction forces. However, we show how to extend our procedures in the presence of Coulomb non-linear friction force acting on the well and to coupled axial-torsional oscillations. Such a characterization of the formation is a new step towards a subsurface-aware drilling system. The first algorithm uses SWD measurements and drill-string dynamics modeling. The algorithm matches the processed direct arrivals of primary and secondary waveforms to the modeled radiation patterns to estimate rocks' seismic velocity while drilling. To model the radiation patterns, we use drill-string dynamics modeling and topdrive measurements to simulate the force-on-bit. The second algorithm is based on parameter estimation techniques applied to the model we have for the bit-rock interaction boundary condition. Such estimation techniques are classical, but we believe that their development in the proposed framework is a novelty. Finally, our last algorithm is based on a machine learning approach. 
Computing the top-drive measured signals' spectra, we train a neural network that estimates the rock's nature. In that sense, our approach is related to the results developed in [? ? ? ]

\subsection{Contributions of this work}

The proposed paper presents a new theoretical framework (which is a novelty in terms of systems theory) that is applied to a complex petroleum engineering problem (estimation of the axial motion of a drilling system and the nature of the rock interacting with the drill-bit). More precisely, the main contributions of this paper are as follows:

- From a mathematical point of view, we develop in this paper a new recursive dynamics interconnection framework. Although this framework is developed here on a drilling application, it can be extended to a large class of balance laws systems [? ]. This framework is a natural environment to develop estimation techniques with a reduced computational cost. We believe it is an important contribution from the system theory perspective.

- From a petroleum engineering point of view, we apply our recursive framework to estimate the axial motion of the drill-string, which is a novelty and represents a step towards automated closed-loop geosteering. The distributed model we use to describe the axial dynamics is based on recent contributions [? ? ] and captures a large part of the possible dynamics for a significantly extended range of well lengths and surveys compared to the more standard lumped approximations. The development of estimation techniques for this class of distributed model is a novelty as most of the contributions in the literature either deal with finite-dimensional approximations of the drilling device (to apply already existing techniques as Kalman's filter [? ]) or develop operational settings to measure the downhole state directly. The long latency ( 1 to $20 \mathrm{~s}$ ) of downhole telemetry has made automatic feedback control significantly slower than manual control [? ? ], which reduces its interest in terms of control application.

- The third contribution consists of using the estimation of the drill-bit source signature to estimate the nature of the rock interacting with the drill-bit. Although the three proposed algorithms are based on existing techniques (SWD, machine-learning, and parameter estimation techniques), their application in this context constitutes an innovation.

- Finally, the last contribution of this paper consists of two extensions on how to adjust the proposed approach to coupled axial-torsional oscillations and the presence of Coulomb friction terms.

Note that the different algorithms are tested in simulations against reliable models. These models are accepted by a large part of the petroleum engineering community as reliable. Extended simulations have been proposed in [? ? ] to test these models and all the trends predicted by the models are supported by field measurements [? ]. However, complete validation of the proposed approach would require several tests against field data. We believe that the development of an experimental setting is outside this paper's scope and would be a contribution by itself.

\subsection{Paper structure and notations}

The paper is organized as follows: In Section 2, we present the mechanical and sensing systems under consideration. In Section 3 , we derive a distributed model that describes the axial dynamics 
of the drill-string. As we consider a multi-sectional drill-string, specific attention is paid to the connections between the different pipes. In Section 4, we express the drill-bit axial force and the drill-bit velocity as functions of the top-drive force and velocity by using an iterative backstepping approach. In Section 5, we present three algorithms to compute the specific intrinsic energy of the drilled rock. The first algorithm uses seismic-while-drilling technique, the second is based on direct parameter estimation, and the third algorithm uses a neural network. Simulation results are given in Section 6. Also, we compare the advantages and drawbacks of each algorithm. Finally, we extend our approach to the case of non-linear Coulomb side-forces and coupled axial-torsional dynamics in Section 7 .

In what follows, given a set $\Omega \subseteq \mathbb{R}$, its characteristic function will be denoted by

$$
\mathbb{1}_{\Omega}(\theta)= \begin{cases}1 & \text { if } \theta \in \Omega \\ 0 & \text { otherwise. }\end{cases}
$$

\section{Mechanical and sensing systems under consideration}

We consider in this paper the mechanical part of a drilling system. A drilling device comprises three components: the surface drill rig, which includes a rotating mechanism (usually a rotary table or a top drive suspended over the drill floor by the traveling block), the drill string, and the Bottom Hole Assembly (BHA). The torsional and axial motions generated at the surface are transferred to the drill string and the BHA. The drill string is an interconnection of pipes that are steel tubes with a length of typically $10 \mathrm{~m}$. These pipes are usually run in tension to avoid the effect of fatigue due to a potential helical buckling. They are hollow so that a mud pump can inject a drilling fluid to clean, cool, and lubricate the bit to evacuate the rock cuttings. The BHA comprises the bit (a rock cutting device), a series of relatively heavy pipe sections, known as drill collars (much thicker pipes that provide the necessary weight to perform the perforation), stabilizers (at least two spaced apart) which prevent the drill string from unbalancing, and "shock subs" that absorb vibrations between the bit and the drill-collars. While the BHA length remains constant, the drill pipes' total length may increase as the borehole depth does. The weight exerted on the bit impacts the cutting process's performance, measured by the Rate-Of-Penetration (ROP). The nature of the boundary conditions at the bit-rock interface is a critical aspect of the model and is discussed in detail below. This mechanical part is combined with a hydraulic system to maintain the Bottom-Hole Circulating Pressure (BHCP) between pre-specified constraints. Such a drilling system is schematically pictured in Figure 1.

Moreover, interactions between the drill bit and rock generate significant elastic energy. Understanding these interactions and modeling the seismic radiation patterns are crucial in improving the drilling performances [? ]. Drill bit-rock interactions generate compressional $(\mathrm{P})$ and vertical components of shear (SV) waves. These waves propagate through the sub-surface and can reach the sensing devices (geophones or hydrophones). In onshore environments, geophones record the particle velocity, and hydrophones, in offshore environments, measure the pressure component. The drill bit-rock interaction wavefields recorded by the sensors near the surface are known as SWD data. Drill bit-rock interactions can also generate reflected wavefields that travel through the drill string. These wavefields can be recorded by accelerometers mounted on top-drive. The accelerometers provide hook-load and hook-speed measurements. The sensing tools give vital information about the sub-surface, which helps us efficiently model the drill string dynamics. 


\section{Parameters}

Table 1: Nomenclature

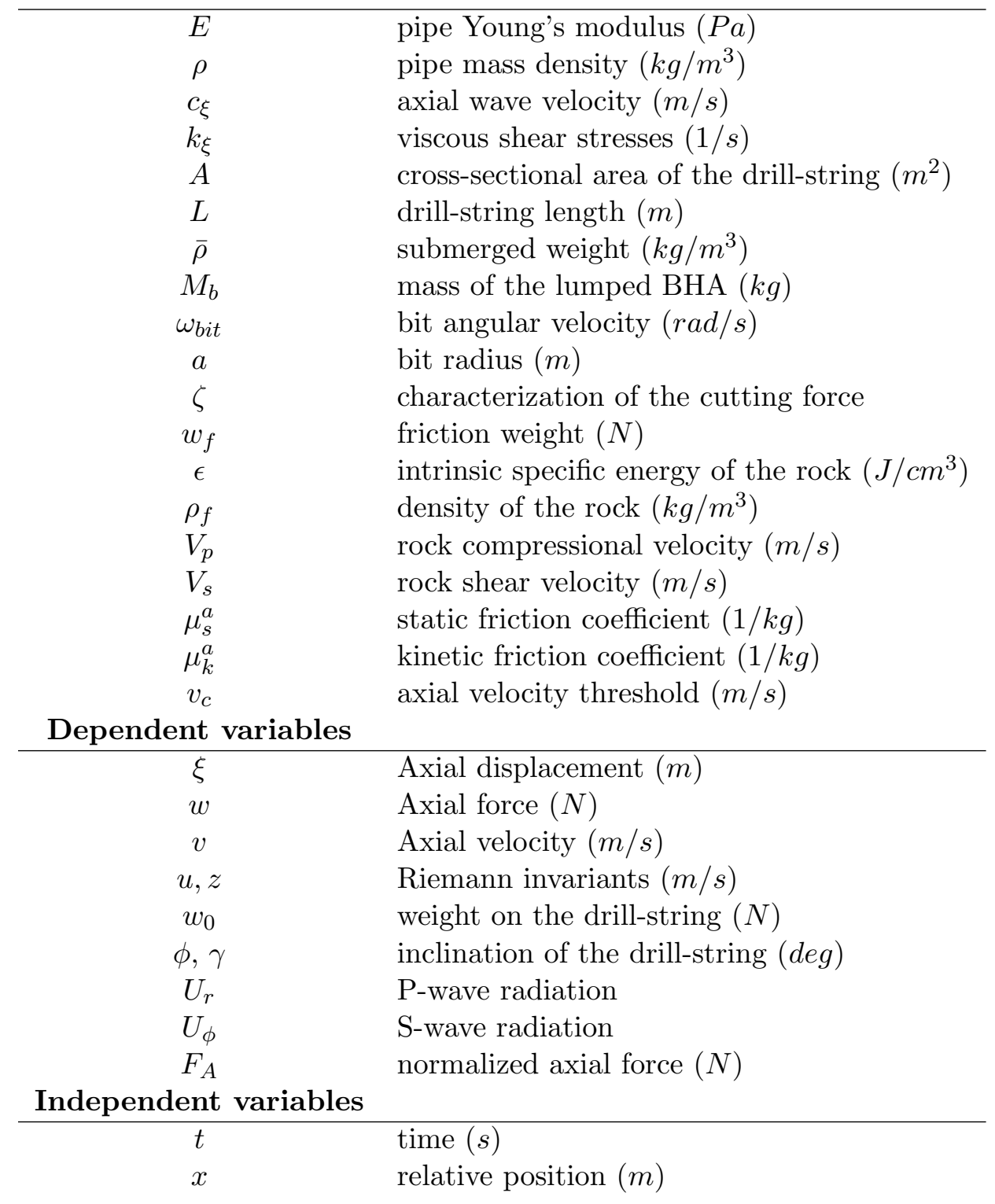




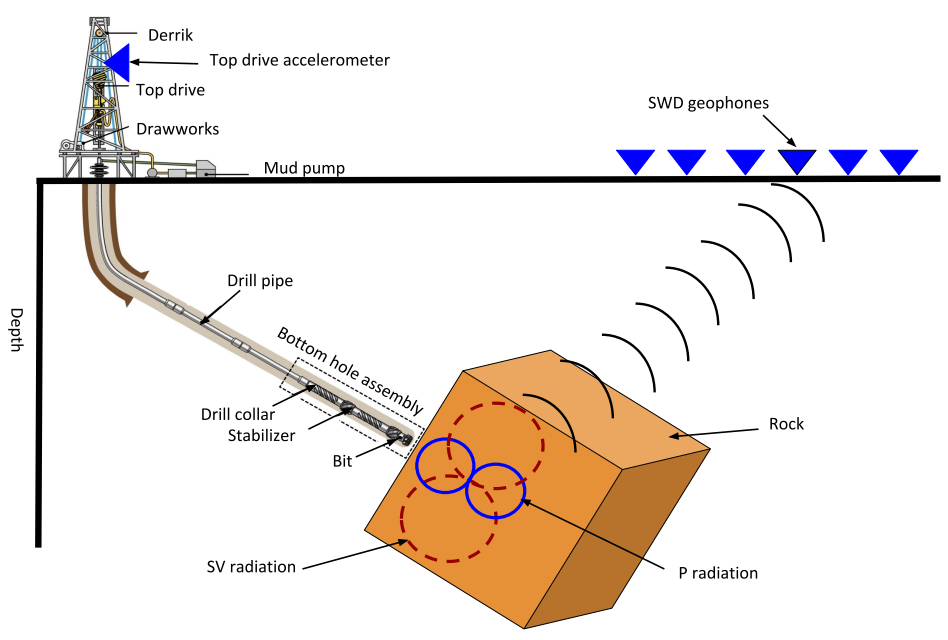

Figure 1: Schematic representation of the drilling and sensing system (Figure modified from [?]).

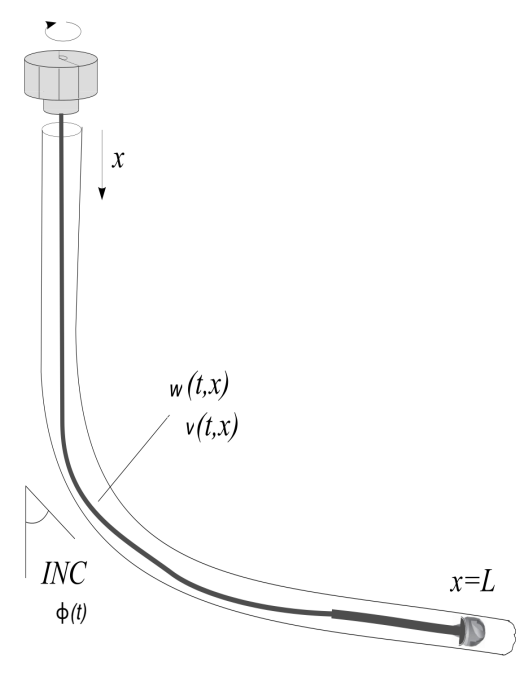

Figure 2: Schematic indicating the distributed drill string lying in deviate borehole.

\section{Drill-string model}

In this section, we present a model that describes the mechanical dynamics of the drill-string. This model is similar to the one presented in [? ] and is inspired by [? ? ]. A flowchart of the mathematical representation of the system is given in Figure 5 at the end of the section.

\subsection{Distributed Axial dynamics of the drill-string}

We model the dynamics of a directional drilling system of length $L$ (Figure 2). In this model, $x$ denotes the curvilinear abscissa, $x=0$ is the top-drive position, and $x=L$ is the position of the drill bit. We denote $\phi(x)$ the well's inclination at the position $x$. Let us denote $\xi(t, x)$ the axial displacement of the drill-string. It is a function of $(t, x)$ evolving in $\{(t, x) \mid 0<t<T, x \in[0, L]\}$ (where $T$ is a positive time). The axial force associated to $\xi$ can be found from the strain, given as the local relative compression:

$$
w(t, x)=A E \frac{(\xi(t, x)-\xi(t, x+d x))}{d x},
$$

$A$ being the cross-sectional area of the drill-string, $E$ being its Young's modulus and $d x \rightarrow 0$ the infinitesimal axial position increment. The axial velocity satisfies

$$
v(t, x)=\frac{\partial \xi(t, x)}{\partial t}
$$

These states are pictured in Figure 2. The ratio diameter/length of a drillstring, typically less than $10^{-4}$, implies that the drillstring can be modeled using an Euler-Bernoulli beam model. More precisely, using the distributed model given in [? ? ], we can derive the dynamics of interest 
by assuming elastic deformations and using equations of continuity and state. The axial motion satisfies the following wave Partial Differential Equation

$$
\frac{\partial^{2} \xi}{\partial t^{2}}(t, x)-c_{\xi}^{2} \frac{\partial^{2} \xi}{\partial x^{2}}(t, x)=-k_{\xi} \frac{\partial \xi}{\partial t}(t, x)+\frac{\bar{\rho}}{\rho} g \sin (\phi(x)),
$$

where $c_{\xi}=\sqrt{\frac{E}{\rho}}, \rho$ being the pipe mass density and $k_{\xi}$ is a damping coefficient representing the viscous shear stresses acting on the pipe. The term $h(x)=\frac{\bar{\rho}}{\rho} g \sin (\phi(x))$ accounts for the acceleration of gravity acting on the submerged weight $\bar{\rho}$. It is a simple (but still realistic and consistent) model for the gravitational force. We neglect for the moment the friction between the drill-string and the borehole. From (2), we have that the axial force and velocity satisfy the following set of PDEs

$$
\begin{aligned}
& \frac{\partial w(t, x)}{\partial t}+A E \frac{\partial v(t, x)}{\partial x}=0, \\
& \frac{\partial v(t, x)}{\partial t}+\frac{1}{A \rho} \frac{\partial w(t, x)}{\partial x}=-k_{\xi} v(t, x)+h(x) .
\end{aligned}
$$

The topside weight on the drill-string, $w(t, 0)$, corresponds to the system actuation. The downhole boundary condition at $x=L$ is obtained from a force balance on the lumped Bottom-Hole Assembly (BHA). These two boundary conditions will be introduced below. Model (3)-(4) does not consider the Coulomb friction between the drill string and the borehole, also known as the side force. It has been shown in [? ] that for torsional oscillations, the normal component of this Coulomb friction term (usually modeled using a differential inclusion) can have significant effects on stability, even when the bit is off-bottom since it can be a source of stick-slip. Consequently, the axial components of such Coulomb non-linear friction terms may also have a non-negligible effect on the dynamics (even if this has not been broadly studied in the literature). We choose not to include this differential inclusion into our model to simplify the design of our different algorithms. However, we will show in Section 7.1 that our procedures can be extended in the presence of such a non-linear term. Note that the proposed model has been extensively analyzed in [? ? ]. All the trends predicted by the models are supported by field measurements [? ]. Moreover, validation against field data (at least for the torsional part of the model) has been proposed in [? ].

\subsection{Discontinuities of a multiple sectioned drill-string}

The lower part of the drill-string is usually made up of drill collars that may have a great impact on the global dynamics due to their inertia [? ]. In particular, these pipes may have different lengths, density, inertia or Young's modulus. This change of the characteristic line impedance may cause reflections in the traveling waves. Let us assume we have $N$ different sections $(N \in \mathbb{N})$, and let us denote $x_{i}$ the spatial coordinate of the junction point between the $(i-1)^{\text {th }}$-section and the $i^{\text {th }}$-section. Let us denote $x_{1}=0, x_{N+1}=L$ and $\left(w^{i}(t, x), v^{i}(t, x)\right)$ the force and velocity along the $i^{\text {th }}$ section of the drill-string. The corresponding physical parameters will also be expressed using the superscript ${ }^{i}$ (for instance $\rho^{i}$ will be the density of the $i^{\text {th }}$ section). The boundary conditions at the transition are given by the following continuity constraints

$$
v^{i}\left(t, x_{i+1}\right)=v^{i+1}\left(t, x_{i+1}\right), \quad w^{i}\left(t, x_{i+1}\right)=w^{i+1}\left(t, x_{i+1}\right) .
$$

When there is no ambiguity, this superscript will be omitted to ease the notations. 


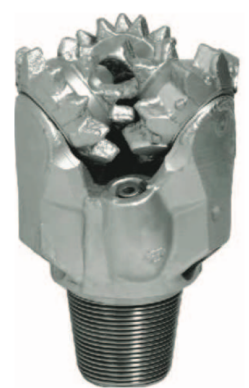

(a)

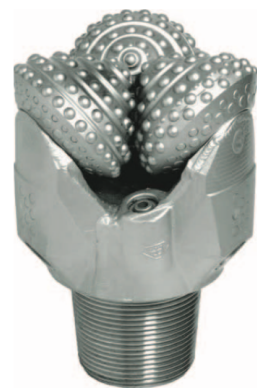

(b)
Figure 3: Roller-cone bits used in softer and shallower formations (a); roller-cone bits used in deeper and harder formations (b) (Source: Adapted from [? ] ).
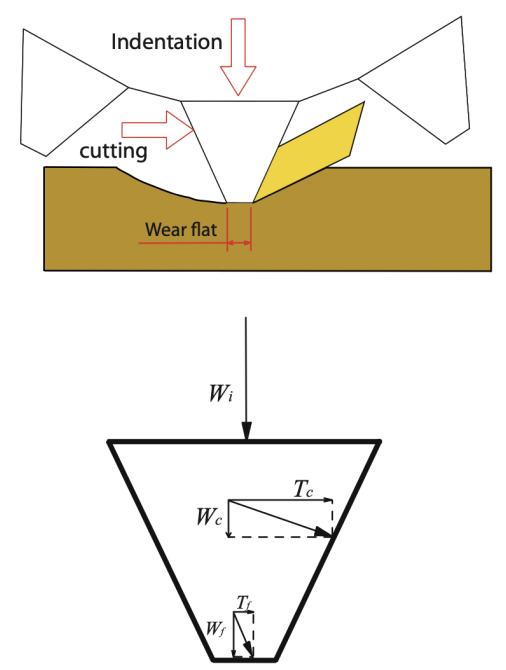

Figure 4: Forces acting on an inverted V shape tooth (Source: Adapted from [?]).

\subsection{Downhole boundary condition: bit-rock interaction}

In this section, we compute the downhole boundary condition. As the length of the BHA $(\approx 200 \mathrm{~m})$ is much smaller than the one of the drill-string $(\approx 2000 \mathrm{~m})$, its effect can be lumped into an ODE coupled with the drill-string [? ? ]. Thus, the downhole boundary condition at $x=L$ can be obtained from a force balance on the lumped BHA. This yields

$$
M_{b} \frac{\partial v}{\partial t}(t, L)=-w_{b}(v(t, L), w(t, L))+w(t, L)+\frac{\bar{\rho}}{\rho} M_{b} g,
$$

where $M_{b}$ is the mass of the lumped BHA and $w_{b}(\cdot, \cdot)$ the force acting from the rock on the BHA through the drilling bit, known as the weight on bit (WOB).

This force balance requires the expression of the WOB. We consider a roller-cone bit. To express the bit-rock interface laws, we can follow the approach developed in [? ] for drag bits (consistent with laboratory results). As suggested in [?], the tool's action is decomposed into three independent processes: a pure cutting process taking place ahead of the cutting face, a pure indentation process, and a frictional contact process mobilized along with the interface between 


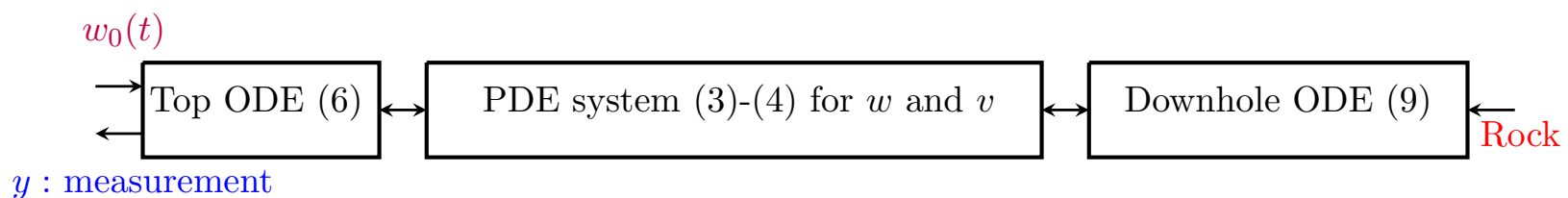

Figure 5: Flowchart of the mathematical model of the drilling device.

the wear flat and the rock. Integrating the effects of all the individual cutters, the weight-on-bit $w_{b}$ can be decomposed into a contribution associated with the forces transmitted by the cutting face of each cutter $\left(w_{c}\right)$, a contribution corresponding to the indentation process $\left(w_{i}\right)$ and another corresponding to the forces acting on the cutter wear flats $\left(w_{f}\right)$. In what follows, we choose to include the contribution $w_{i}$ into $w_{f}$ since both are constant. These two forces are pictured in Figure 4. Following the approach elaborated within the context of PDC bits [? ? ], the cutting process is split into three successive regimes. A frictional contact process dominates the first phase (Phase I) due to bit dullness. During the second phase (Phase II), contact forces are fully mobilized, and additional forces contribute to cutting. Finally, during the last phase (Phase III), the frictional contact forces increase due to insufficient hole cleaning. Ideally, drilling should be performed to the foundering point (Phase II/III transition). As shown in [? ], the expression of the WOB depends on the phase. We will assume that we are in Phase II (cutting phase) and that the depth of cut is greater than the combined depth of cut at Phase II/III transition. Consequently, the WOB is expressed as

$$
w_{b}(v(t, L), w(t, L))=w_{f}+w_{c}=w_{f}+K d(t)=w_{f}+a \zeta \epsilon d(t),
$$

where $w_{f}$ is a friction weight independent of the bit velocity (and therefore constant) while $K=a \zeta \epsilon$, with $a$ being the bit radius, $\zeta$ a characterization of the cutting angle and $\epsilon$ the intrinsic specific energy of the rock [? ? ]. The function $d(t)$ is the combined depth of cut per revolution [? ? ? ]. It satisfies the following equation,

$$
d(t)=\frac{v(t, L)}{\omega_{\mathrm{bit}}(t)},
$$

where the bit angular velocity $\omega_{\text {bit }}$ is assumed known here. We have previously presented a fully validated torsional model to estimate $\omega_{\text {bit }}$ which may be integrated into the present here through a coupling at the drill-bit [? ]. More details about axial-torsional couplings are given in Section 7.2. Experimental evidence for the proposed expression of the weight-on-bit (equation (8)) for drag bits have been obtained with a small drilling machine in [? ]. For a roller-cone bit, such an expression has been validated in [? ] with series of laboratory tests at atmospheric pressure conducted with an in-house designed drilling rig together with published experimental data.

Thus, the boundary condition (7) rewrites as

$$
M_{b} \frac{\partial^{2} \xi}{\partial t^{2}}(t, L)=-\frac{a \zeta \epsilon}{\omega_{\mathrm{bit}}} \frac{\partial \xi(t, L)}{\partial t}-w_{f}-E A \frac{\partial \xi(t, L)}{\partial x}+\frac{\bar{\rho}}{\rho} M_{b} g .
$$

\subsection{Problem under consideration}

The present paper's objective is to provide a near real-time estimation of the nature of the drilled rock, i.e., intrinsic energy $\epsilon$ or seismic velocity, to improve drill-string dynamics estimation. 
To properly model the drill-string dynamics, we need to know the seismic velocity of the drilled rocks. However, before drilling, the seismic velocities provided by surface seismic processing may be inaccurate. Here, we extend the results of [? ] to the case of a non-vertical multi-sectionals drill-string. Moreover, we present two new algorithms for estimating the seismic velocities of rocks. The developed algorithms only require knowledge of the physical properties of the drilling system:

1. The first algorithm uses seismic-while-drilling measurements and extends the methodology of [? ]. In this procedure, we first express the force exerted on the bit as a function of the (measured) top-drive force and velocity without depending on the downhole boundary condition or on the subsurface. We estimate the rock's seismic velocity by combining this expression with the knowledge of far-field radial and angular displacements (obtained by using seismic sensors).

2. The second algorithm directly uses the boundary condition (9). We express the force exerted on the bit and the corresponding velocity as functions of the (measured) top-drive force and velocity. Then, we apply signal filtering and parameters estimation techniques to equation (9) to obtain the intrinsic specific energy of the rock.

3. The last algorithm is based on a machine learning approach. Applying a Fast Fourier Transform (FFT) to the measured outputs (top-drive force and velocity) and choosing relevant attributes such as the dominant frequencies and their corresponding gains. Then, by pairing the relevant attributes to their corresponding $\epsilon$, we run a neural network learning algorithm to train the model. The training is done with a set of thousand simulations for which the parameters of interest are modified between each run.

Also, we discuss the advantages and drawbacks of each procedure. Moreover, we show how the proposed algorithms can be extended in the presence of non-linear Coulomb friction terms (see Section 7.1) and to systems with coupled axial-torsional oscillations (see Section 7.2).

\section{Expression of the drill-bit axial force and velocity}

Among the three algorithms we introduce in this paper, two of them require the knowledge of the axial force and velocity exerted at the drill-bit. It has been shown in [? ] that it is possible (for a vertical uni-sectional well) to express the drill-bit source signature as a function of top-drive force and velocity measurements. The proposed method was based on the Riemann invariants and the backstepping methodology. Here, we extend the results of [? ] to the case of our multi-sectional non-vertical well.

\subsection{Derivation of Riemann invariants}

The Riemann invariants of a hyperbolic PDE are the states corresponding to a transformation of the system which has a diagonalized transport matrix, i.e. the system can be written as a series of transport equations only coupled through the source terms [? ]. On the set $\{(t, x) \mid 0<t<$ $\left.T, \quad x \in\left[x_{i}, x_{i+1}\right]\right\}$, we define the Riemann invariants as

$$
u^{i}(t, x)=\left(\frac{\partial}{\partial t} \xi^{i}(t, x)-c_{\xi}^{i} \frac{\partial}{\partial x} \xi^{i}(t, x)\right) \mathrm{e}^{\frac{k_{\xi}^{i}}{2 c_{\xi}^{i}} x}, z^{i}(t, x)=\left(\frac{\partial}{\partial t} \xi^{i}(t, x)+c_{\xi}^{i} \frac{\partial}{\partial x} \xi^{i}(t, x)\right) \mathrm{e}^{-\frac{k_{\xi}^{i}}{2 c_{\xi}^{i}} x} .
$$


Consequently, on each section, equation 2 rewrites

$$
\begin{gathered}
\frac{\partial}{\partial t} u^{i}(t, x)+c_{\xi}^{i} \frac{\partial}{\partial x} u^{i}(t, x)=-\frac{k_{\xi}^{i}}{2} \mathrm{e}^{\frac{k_{\xi}^{i}}{c_{\xi}^{i}} x} z^{i}(t, x)+h_{1}^{i}(x), \\
\frac{\partial}{\partial t} z^{i}(t, x)-c_{\xi}^{i} \frac{\partial}{\partial x} z^{i}(t, x)=-\frac{k_{\xi}^{i}}{2} \mathrm{e}^{-\frac{k_{\xi}^{i}}{c_{\xi}^{i}} x} u^{i}(t, x)+h_{2}^{i}(x),
\end{gathered}
$$

where $h_{1}^{i}(x)=h(x) \mathrm{e}^{\frac{k_{\xi}^{i}}{2 c_{\xi}^{i}} x}$ and $h_{2}^{i}(x)=h(x) \mathrm{e}^{-\frac{k_{\xi}^{i}}{2 c_{\xi}^{i}} x}$. In the Riemann coordinates, the boundary conditions at the junctions (5) rewrite for $i<N+1$

$$
\begin{aligned}
z^{i}\left(t, x_{i+1}\right) & =a_{1}^{i} u^{i}\left(t, x_{i+1}\right)+a_{2}^{i} z^{i+1}\left(t, x_{i+1}\right), \\
u^{i+1}\left(t, x_{i+1}\right) & =a_{3}^{i} u^{i}\left(t, x_{i+1}\right)+a_{4}^{i} z^{i+1}\left(t, x_{i+1}\right),
\end{aligned}
$$

where

$$
a_{1}^{i}=\frac{1-Z^{i}}{1+Z^{i}} \mathrm{e}^{-\frac{k_{\xi}^{i}}{c_{\xi}^{i}} x_{i+1}}, a_{2}^{i}=\frac{2 Z^{i}}{1+Z^{i}} \mathrm{e}^{\left(\frac{k_{\xi}^{i+1}}{2 c_{\xi}^{i+1}}-\frac{k_{\xi}^{i}}{2 c_{\xi}^{i}}\right) x_{i+1}}, a_{3}^{i}=\frac{1}{Z^{i}} a_{2}^{i}, a_{4}^{i}=\frac{Z^{i}-1}{1+Z^{i}} \mathrm{e}^{\frac{k_{\xi}^{i+1}}{c_{\xi}^{+1+1}} x_{i+1}},
$$

where we have denoted the relative magnitude of the impedance as

$$
Z^{i}=\frac{c_{\xi}^{i}}{E^{i} A^{i}} / \frac{c_{\xi}^{i+1}}{E^{i+1} A^{i+1}}
$$

The boundary condition (6) remains unchanged

$$
u^{1}(t, 0)=z^{1}(t, 0)+\frac{2 c_{\xi}^{1}}{E^{1} A^{1}} w_{0}(t),
$$

while the boundary condition 99 rewrites

$$
\begin{aligned}
& z(t, L)=-\mathrm{e}^{-\frac{k_{\xi}}{c_{\xi}} L} u(t, L)+2 \mathrm{e}^{-\frac{k_{\xi}}{2 c_{\xi}} L} X(t), \\
& \dot{X}(t)=-\frac{a \zeta \epsilon}{M_{b} \omega_{\mathrm{bit}}} X(t)-\frac{w_{f}}{M_{b}}+\frac{\bar{\rho}}{\rho} g-\frac{E A_{s}}{2 c_{\xi} M_{b}}\left(z(t, L) \mathrm{e}^{\frac{k_{\xi}}{2 c_{\xi}} L}-u(t, L) \mathrm{e}^{-\frac{k_{\xi}}{2 c_{\xi}} L}\right) .
\end{aligned}
$$

\subsection{Delayed equations}

In this section, we adjust the ideas of [? ] to the case of our non-vertical well with multiple connections. The objective is to express our PDEs system as delayed equations. Such a delay approach is particularly suitable for this kind of systems due to the transport structure. It allows using the wide range of tools already developed for time-delay systems. Let us consider the extension for the backstepping Volterra transformation [? ? ] that has been introduced in [? ]

$$
\begin{aligned}
& \alpha^{i}(t, x)=u^{i}(t, x)+\int_{x_{i}}^{x}\left(K_{i}^{u u}(x, y) u^{i}(t, y)+K_{i}^{u z}(x, y) z^{i}(t, y)\right) d y, \\
& \beta^{i}(t, x)=z^{i}(t, x)+\int_{x_{i}}^{x}\left(K_{i}^{z u}(x, y) u^{i}(t, y)+K_{i}^{z z}(x, y) z^{i}(t, y)\right) d y,
\end{aligned}
$$


where the kernels $K_{i}^{u u}, K_{i}^{z u}, K_{i}^{u z}$ and $K_{i}^{z z}$ are continuous functions defined on the domain $\mathcal{T}_{i}=$ $\left\{(x, y) \in\left[x_{i}, x_{i+1}\right]^{2}, y \leq x\right\}$. They satisfy a set of PDEs (that admits a unique solution) that is given in [?] (replacing 0 by $x_{i}$ ). Note that theses kernels can be explicitly computed following the approach given in [?]

Differentiating (20)-21) with respect to time and space and integrating by parts, one can show that the states $\alpha^{i}$ and $\beta^{i}$ satisfy the following set of transport PDEs

$$
\begin{aligned}
\frac{\partial}{\partial t} \alpha^{i}(t, x)+c_{\xi}^{i} \frac{\partial}{\partial x} \alpha^{i}(t, x) & =-c_{\xi}^{i} K_{i}^{u z}\left(x, x_{i}\right) \beta^{i}\left(t, x_{i}\right)+h_{3}^{i}(x), \\
\frac{\partial}{\partial t} \beta^{i}(t, x)-c_{\xi}^{i} \frac{\partial}{\partial x} \beta^{i}(t, x) & =c_{\xi}^{i} K_{i}^{z u}\left(x, x_{i}\right) \alpha^{i}\left(t, x_{i}\right)+h_{4}^{i}(x) .
\end{aligned}
$$

where

$$
\begin{aligned}
& h_{3}^{i}(x)=h_{1}^{i}(x)+\int_{x_{i}}^{x} K_{i}^{u u}(x, y) h_{1}(y)+K_{i}^{u v}(x, y) h_{2}(y) d y, \\
& h_{4}^{i}(x)=h_{2}^{i}(x)+\int_{x_{i}}^{x} K_{i}^{v u}(x, y) h_{1}(y)+K_{i}^{v v}(x, y) h_{2}(y) d y .
\end{aligned}
$$

Applying the method of characteristics on (22)-23], for any $x \in\left[x_{i}, x_{i+1}\right]$ and for any $t \geq \frac{x}{c_{\xi}}$, we get

$$
\begin{aligned}
& \alpha^{i}(t, x)=\alpha^{i}\left(t-\frac{x-x_{i}}{c_{\xi}^{i}}, x_{i}\right)+\int_{0}^{\frac{x-x_{i}}{c_{\xi}^{i}}} h_{3}^{i}\left(x-c_{\xi}^{i} s\right)-c_{\xi}^{i} K_{i}^{u z}\left(x-c_{\xi}^{i} s, x_{i}\right) \beta^{i}\left(t-s, x_{i}\right) d s, \\
& \beta^{i}(t, x)=\beta^{i}\left(t+\frac{x-x_{i}}{c_{\xi}^{i}}, x_{i}\right)-\int_{0}^{\frac{x-x_{i}}{c_{\xi}^{i}}} h_{4}^{i}\left(x-c_{\xi}^{i} s\right)-c_{\xi}^{i} K_{i}^{z u}\left(x-c_{\xi}^{i} s, x_{i}\right) \alpha^{i}\left(t+s, x_{i}\right) d s .
\end{aligned}
$$

Following the path of [? ], we obtain the following expressions (the complete computations are given in Appendix)

$$
\begin{aligned}
w^{i}\left(t, x_{i+1}\right)= & \frac{E^{i} A^{i}}{2 c_{\xi}^{i}}\left(\mathrm{e}^{-\frac{k_{\xi}^{i} \Delta_{i}}{2 c_{\xi}}} v^{i}\left(t-\frac{\Delta_{i}}{c_{\xi}^{i}}, x_{i}\right)-\mathrm{e}^{\frac{k_{\xi}^{i} \Delta_{i}}{2 c_{\xi}^{i}}} v^{i}\left(t+\frac{\Delta_{i}}{c_{\xi}^{i}}, x_{i}\right)\right)+\frac{1}{2}\left(\mathrm{e}^{-\frac{k_{\xi}^{i} \Delta_{i}}{2 c_{\xi}^{i}}} w^{i}\left(t-\frac{\Delta_{i}}{c_{\xi}^{i}}, x_{i}\right)\right. \\
& \left.+\mathrm{e}^{\frac{k_{\xi}^{i} \Delta_{i}}{2 c_{\xi}^{i}}} w^{i}\left(t+\frac{\Delta_{i}}{c_{\xi}^{i}}, x_{i}\right)\right)+\int_{-\frac{\Delta_{i}}{c_{\xi}^{i}}}^{\frac{\Delta_{i}}{c_{\xi}^{i}}}\left(\mathrm{e}^{\frac{k_{\xi}^{i}}{2 c_{\xi}^{i}} x_{i}} f_{u}^{i}(s)+\mathrm{e}^{-\frac{k_{\xi}^{i}}{2 c_{\xi}^{i}} x_{i}} f_{z}^{i}(s)\right) v^{i}\left(t-s, x_{i}\right) d s \\
& +\int_{-\frac{\Delta_{i}}{c_{\xi}^{i}}}^{\frac{\Delta_{i}}{c_{\xi}^{i}}} \frac{c_{\xi}^{i}}{E A}\left(\mathrm{e}^{\frac{k_{\xi}^{i}}{2 c_{\xi}^{i}} x_{i}} f_{u}^{i}(s)-\mathrm{e}^{-\frac{k_{\xi}^{i}}{2 c_{\xi}^{i}} x_{i}} f_{z}^{i}(s)\right) w^{i}\left(t-s, x_{i}\right) d s+G_{w}^{i},
\end{aligned}
$$


and

$$
\begin{aligned}
v^{i}\left(t, x_{i+1}\right)= & \frac{1}{2}\left(\mathrm{e}^{-\frac{k_{\xi}^{i} \Delta_{i}}{2 c_{\xi}}} v^{i}\left(t-\frac{\Delta_{i}}{c_{\xi}^{i}}, x_{i}\right)+\mathrm{e}^{\frac{k_{\xi}^{i} \Delta_{i}}{2 c_{\xi}^{i}}} v^{i}\left(t+\frac{\Delta_{i}}{c_{\xi}^{i}}, x_{i}\right)\right)+\frac{c_{\xi}^{i}}{2 E^{i} A^{i}}\left(\mathrm{e}^{-\frac{k_{\xi}^{i} \Delta_{i}}{2 c_{\xi}^{i}}} w^{i}\left(t-\frac{\Delta_{i}}{c_{\xi}^{i}}, x_{i}\right)\right. \\
& \left.-\mathrm{e}^{\frac{k_{\xi}^{i} \Delta_{i}}{2 c_{\xi}^{i}}} w^{i}\left(t+\frac{\Delta_{i}}{c_{\xi}^{i}}, x_{i}\right)\right)+\int_{-\frac{\Delta_{i}}{c_{\xi}^{i}}}^{\frac{\Delta_{i}}{c_{\xi}^{i}}}\left(g_{u}^{i}(s) \mathrm{e}^{\frac{k_{\xi}^{i}}{c_{\xi}^{i}} x_{i}}+g_{z}^{i}(s) \mathrm{e}^{-\frac{k_{\xi}^{i}}{2 c_{\xi}^{i}} x_{i}}\right) v^{i}\left(t-s, x_{i}\right) d s \\
& +\int_{-\frac{\Delta_{i}}{c_{\xi}^{i}}}^{\frac{\Delta_{i}}{c_{\xi}^{i}}} \frac{c_{\xi}^{i}}{E A}\left(g_{u}^{i}(s) \mathrm{e}^{\frac{k_{\xi}^{i}}{2 c_{\xi}^{i}} x_{i}}-g_{z}^{i}(s) \mathrm{e}^{-\frac{k_{\xi}^{i}}{2 c_{\xi}^{i}} x_{i}}\right) w^{i}\left(t-s, x_{i}\right) d s+G_{z}^{i},
\end{aligned}
$$

where $\Delta_{i}=x_{i+1}-x_{i}$, where $f_{u}^{i}, f_{z}^{i}, g_{u}^{i}$ and $g_{z}^{i}$ are defined by equations (52)-(55) and where

$$
G_{w}^{i}=\frac{E^{i} A^{i}}{2 c_{\xi}^{i}}\left(\mathrm{e}^{-\frac{k_{\xi}^{i}}{2 c_{\xi}^{i}} x_{i+1}} H_{u}^{i}-\mathrm{e}^{\frac{k_{\xi}^{i}}{2 c_{\xi}^{i}} x_{i+1}} H_{z}^{i}\right), \quad G_{z}^{i}=\frac{1}{2}\left(\mathrm{e}^{-\frac{k_{\xi}^{i}}{2 c_{\xi}^{i}} x_{i+1}} H_{u}^{i}+\mathrm{e}^{\frac{k_{\xi}^{i}}{2 c_{\xi}^{i}} x_{i+1}} H_{z}^{i}\right),
$$

the functions $H_{u}^{i}$ and $H_{z}^{i}$ being defined by equations (49) and (51).

\subsection{Expression of the downhole velocity and force}

Equations (26) and (27) allow us to compute the velocity and the force at the point $x=x_{i+1}$ simply knowing past and futures values of these states at the point $x=x_{i}$. Using the continuity of the force and velocity at each junction, we can iterate the procedure and consequently express the downhole velocity and force as functions of past and futures values of the topside states (the total time-delay window being $\left.\left[-\sum_{i=1}^{N} \frac{\Delta_{i}}{c_{\xi}^{i}}, \sum_{i=1}^{N} \frac{\Delta_{i}}{c_{\xi}^{i}}\right]\right)$. Obviously, in the case of a uni-sectional vertical well, this corresponds to [? , Equation (49)]. It is interesting to note that such an expression does not directly depend on the downhole boundary condition. It is now possible to use the drill-bit source signature to estimate the nature of the drilled rock.

\section{Estimation of the specific intrinsic energy $\epsilon$}

The intrinsic specific energy refers to the amount of energy required to cut a unit volume of rock. This value is dependent on cutter geometry, depth of cut, and rock. However, similar to scratch test studies in rock mechanics, we assume that the specific intrinsic energy is a constant quantity characterizing a particular combination of cutter geometry and rock. This section presents three different algorithms to estimate the parameter $\epsilon$ and discuss their respective advantages and drawbacks. Two of these procedures require the knowledge of the drill-bit source signature. We use the drill-bit source signature estimation methodology in these algorithms, which is presented in the previous section. The different algorithms are summarized by Algorithms 1, 2 and 3 .

\subsection{Wavelet-based approaches}

The two first algorithms we present are based on the knowledge of the drill-bit source signature. The first one combines the drill-bit force and velocity estimation with Seismic While Drilling techniques, while the second one estimates the parameter $\epsilon$ directly from the model. 


\subsubsection{Seismic While Drilling estimation (Algo. 1)}

This section shows how to estimate intrinsic specific energy for different rocks using seismicwhile-drilling (SWD) data. Richard and Dagrain [? ], by using different rock types, study the relationship between the parameter $\epsilon$ and the Uniaxial Compressive Strength (UCS). They show that by expressing the $\epsilon$ as stress rather than energy, the parameter $\epsilon$ is correlated with UCS

$$
\epsilon(M P a) \approx U C S(M P a) .
$$

Several recent works aim to relate the rock strength to the seismic velocity of primary wave [? ]. For example, by comprehensive analysis of different rock types, ranging from sedimentary to metamorphic, Sharma and Singh [? ] show that the seismic velocity of the primary wave and UCS are linearly correlated, and the relationship is as follows

$$
U C S=0.0642 V_{p}-117.99,
$$

where $V_{p}(\mathrm{~m} / \mathrm{s})$ is the seismic velocity of the primary wave. This relationship is derived based on linear regression, and a strong correlation of $R^{2}=0.9022$ is reported. By plugging equation (30) into equation $(29)$, we get

$$
\epsilon \approx 0.0642 V_{p}-117.99 \text {. }
$$

Hence, by knowing the rock's seismic velocity that the system is drilling into, the estimation of $\epsilon$ is possible. Next, we show how to estimate the velocities of rocks while drilling.

SWD measurements record the radiated elastic energy from the drill-bit rock interaction that is traveled through the earth's structure. For example, variations in the amplitude of P-waves and S-waves' direct arrivals in the processed SWD measurements, after removing the source signature, reflect the changes in the rock properties near the drill-bit. In other words, changes in the energy of P-waves and S-waves' direct arrivals can imply that the bit is turning right and that the well's trajectory is modified. The direct arrivals can be used in a relative sense to infer the relative changes in the rock properties such as their seismic velocities or unconstrained rock strengths [? ]. However, Rector and Hardage [? ] show that the radiation patterns of the direct arrivals depend on the $V_{p}$ and $V_{s}$ velocities of the rock interacting with the drill-bit.

In the deviated well with an inclination angle of $\gamma$, the radiation pattern for the primary wave is as follows

$$
U_{r_{j}}\left(r_{j}, \phi_{j}, t\right)=\frac{A_{1} \cos \left(\phi_{j}+\gamma\right)}{\rho_{f} V_{p}^{2} r_{j}} \hat{w}\left(t-\frac{r_{j}}{V_{p}}, L\right),
$$

where $r_{j}$ is the distance from drill-bit to the $j^{t h}$ receiver near the surface, $\phi_{j}$ is the opening angle between the drill-bit and $j^{\text {th }}$ receiver, measured relative to $z$ axis, $\gamma$ is an inclination angle of drill-string, which is measured as a deviation angle with respect to $z$ axis, $t$ is time, $L$ is the length of drill-string, $\rho_{f}$ is the density of the interacting rock with the drill-bit, $\hat{w}=w \odot w$ is the auto-correlation of drill-bit source signature, $A_{1}$ is a constant, and $U_{r_{j}}$ is the far field radial component of the primary wave radiation pattern recorded by the $j^{\text {th }}$ receiver. In most rocks we have $\rho_{f}=1.74 V_{p}^{0.25}$, hence equation (30) simplifies to

$$
U_{r_{j}}\left(r_{j}, \phi_{j}, t\right)=\frac{A_{1} \cos \left(\phi_{j}+\gamma\right)}{1.74 V_{p}^{2.25} r_{j}} \hat{w}\left(t-\frac{r_{j}}{V_{p}}, L\right) .
$$

Similarly, the far-field radiation pattern for secondary wave reads

$$
U_{\phi_{j}}\left(r_{j}, \phi_{j}, t\right)=\frac{A_{1} \sin \left(\phi_{j}+\gamma\right)}{1.74 V_{p}^{0.25} V_{s}^{2} r_{j}} \hat{w}\left(t-\frac{r_{j}}{V_{s}}, L\right) .
$$


By matching the direct arrivals of the primary wave in the source compensated SWD data to the radiation pattern in equation (33), we can estimate the primary velocity of rock interacting with the drill-bit. However, there are three unknowns in equation (33), i.e., $V_{p}, A_{1}$, and $w$. Hence, first, we need to estimate the drill-bit source signature. Using equation (26) and the iterative approach described in Section 4.3, we can consider as available the (delayed) values of the downhole force, i.e., drill-bit source signature. Now that we have the estimate of the drill-bit source signature $w$, the only unknowns in equation (33) are the scaling factor $A_{1}$, and the primary velocity of rock $V_{p}$. The cross-correlation of the estimated source signature with the SWD data gives the primary and secondary direct arrivals. By least-squares matching of the primary direct arrivals in the source compensated SWD data, $U_{r_{j}}^{\text {obs }}\left(r_{j}, \phi_{j}, t\right)$, with the radiation pattern represented in equation (33) and after plugging the auto-correlation of the estimated source signature, we estimate the primary wave velocity of the rock by minimizing the following cost function

$$
\left\{\hat{A}_{1}, \hat{V}_{p}\right\}=\underset{A_{1}, V_{p}}{\operatorname{argmin}} \sum_{i=1}^{N} \sum_{j=1}^{M}\left(U_{r_{j}}^{o b s}\left(r_{j}, \phi_{j}, t_{i}\right)-U_{r_{j}}^{c a l}\left(r_{j}, \phi_{j}, t_{i}\right)\right)^{2},
$$

where $U_{r_{j}}^{c a l}$ is the radiation pattern of primary wave estimated by using equation (33), $N$ is the number of time samples in the data, and $M$ is the number of receivers or channels that are recoding the SWD data. Equation (35) is solved by the least-squares matching method. Finally, after estimating the primary wave velocity of rock, we estimate $\epsilon$ using (31).

After solving for $V_{p}$, we can also apply the same procedure to estimate the secondary velocity of rocks $V_{s}$. To do so, we minimize

$$
\hat{V}_{s}=\underset{V_{s}}{\operatorname{argmin}} \sum_{i=1}^{N} \sum_{j=1}^{M}\left(U_{\phi_{j}}^{o b s}\left(r_{j}, \phi_{j}, t_{i}\right)-U_{\phi_{j}}^{c a l}\left(r_{j}, \phi_{j}, t_{i}\right)\right)^{2} .
$$

Equation (36) is solved by the least-squares matching method, as well. Note that, after solving equation (35), the values of $V_{p}$, and $A_{1}$ are known. So, by plugging the estimates of $V_{p}, A_{1}$, and the auto-correlation of drill-bit source signature into the cost function of equation (36), the only un-known is $V_{s}$. By having the estimates of $V_{p}$, and $V_{s}$, the $\frac{V_{p}}{V_{s}}$ ratio can be further used to identify reservoir fluids [?]. The algorithm is described by Algorithm 1. Although this algorithm requires several seismic sensors, the corresponding advantage is an increased robustness.

\subsubsection{Direct estimation from equation (9) (Algo. 2)}

The specific intrinsic energy of the rock naturally appears in equation (9). Using equations (26)(27) and the iterative approach described in Section 4.3, we can consider as available the (delayed) values of the downhole force and velocity. Regarding the term $\frac{\partial^{2} \xi}{\partial t^{2}}(t, L)$ (that corresponds to $\left.\frac{\partial v}{\partial t}(t, L)\right)$ that appears in equation (9), we can obtain it by differentiating the estimated downhole velocity $v(t, L)$. Of course, the corresponding signals should be low-pass filtered to minimize the negative effects of the boosted high frequency noise. Equation (9) rewrites as

$$
\psi(t)=\epsilon \phi(t)+C,
$$

where $\phi$ and $\psi$ are known functions defined by

$$
\psi(t)=M_{b} \frac{\partial v}{\partial t}(t, L)-w(t, L), \quad \phi(t)=\frac{a \zeta}{\omega_{\mathrm{bit}}(t)} v(t),
$$




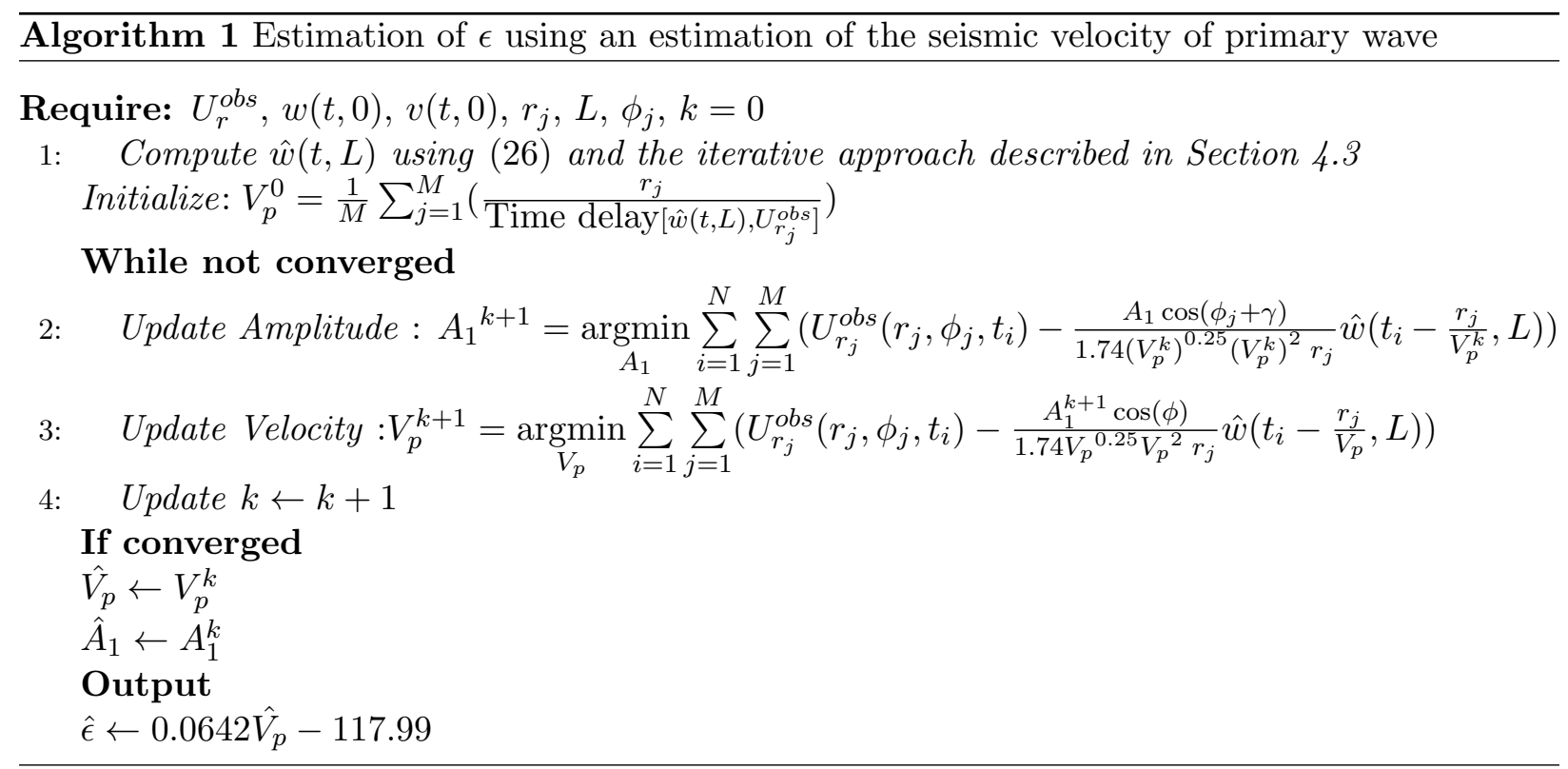

and where $C$ is a constant that is defined by

$$
C=-w_{f}+\frac{\bar{\rho}}{\rho} M_{b} g
$$

Note that the term $C$ is potentially unknown (due to the term $w_{f}$ ). Then, it becomes possible to use parameter estimation techniques (such as Recursive Least Squares) to estimate the unknown parameters $\epsilon$ and $B$. More precisely, these parameters are estimated by minimizing the following cost function

$$
\{\hat{\epsilon}, \hat{C}\}=\underset{\epsilon, C}{\operatorname{argmin}} \sum_{i=1}^{N}\left(\hat{\psi}\left(t_{i}\right)-\epsilon \hat{\phi}\left(t_{i}\right)-C\right)^{2},
$$

where $\hat{\phi}$ and $\hat{\psi}$ are the estimations of the functions $\phi$ and $\psi$ obtained using the iterative approach described in Section 4.3. It is worth mentioning that with this procedure the gravitational forces (i.e. the functions $G_{w}^{i}$ and $G_{z}^{i}$ that appear in equations (26) and (27p) do not actually need to be estimated since these constant terms could be embedded into the term $C$. The main advantage of this approach is that it is easy to implement and only requires top-drive measurements and the knowledge of the different physical parameters of the well. However, such a procedure may be sensitive to noise or to sensor defaults. To reduce the noise negative effects, we can filter the measured signals with a low-pass filter (Butterworth filter for instance). Finally, this procedure strongly depends on the accuracy of our model to describe the bit-rock interaction. The algorithm is summarized by Algorithm 2

\subsection{Machine Learning estimation (Algo. 3)}

The last procedure we present in this paper is based on Machine Learning ([? ]). It does not require any specific knowledge of the system (no model is needed). Machine learning algorithms have already be used in control applications in [? ] or in [? ], using adaptive neural networks. Since the measured outputs (top-drive force and velocity) somehow depend on the rock's intrinsic 


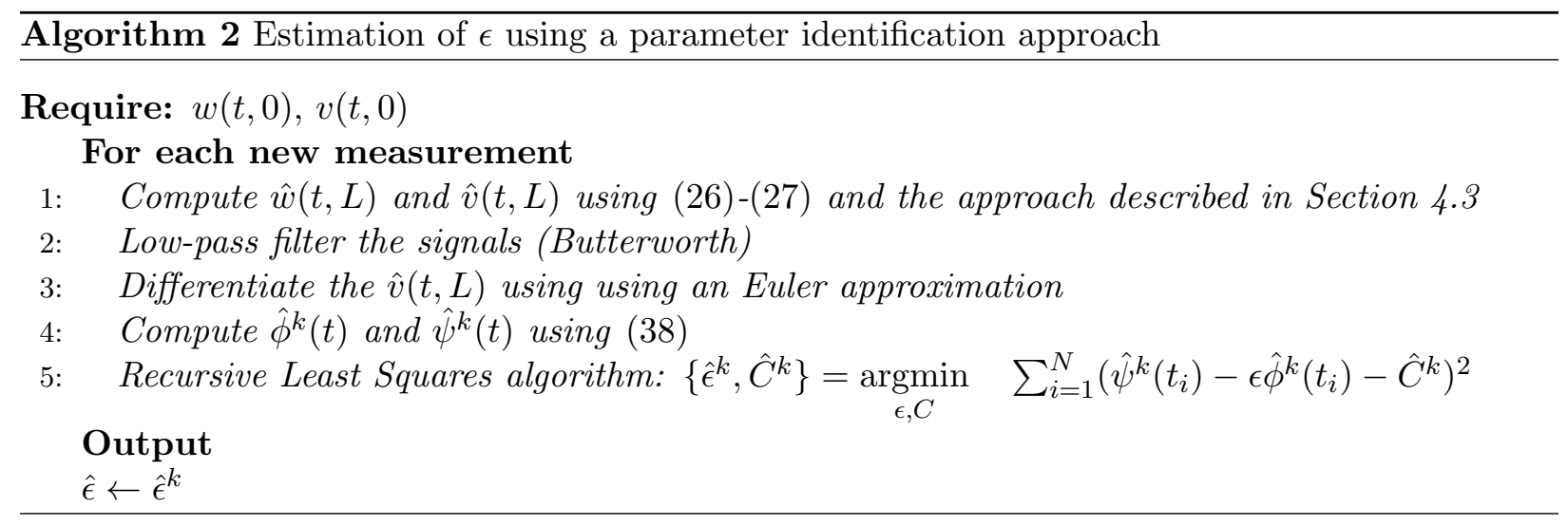

specific energy, their spectrum should contain sufficient information to distinguish the drilled rock's nature. By applying a Fast Fourier Transform (FFT) to the measured outputs (top-drive force and velocity), we can obtain these signals' spectra. More precisely, let us denote $y(t)$ the measured continuous output. In practice, this signal is not continuous but sampled (with a sampling rate that depends on our sensors' quality). Let us denote $\mathrm{Y}$, the vector obtained by concatenation of all our measurements. Let us assume $Y$ has $N$ components (i.e., we have $N$ measurements). Its discrete Fourier transform $\hat{Y}$ is a vector with $N$ components defined by

$$
\hat{Y}(k)=\sum_{n=1}^{N} y(k) \mathrm{e}^{-2 \pi i * \frac{(k-1)(n-1)}{N}} .
$$

The vector $\hat{Y}$ is a complex function that corresponds to the spectrum of the signal $y$. Several attributes characterize it. Among them, we can cite the dominant peak (highest value of the modulus of $\hat{Y}$ ), the number of peaks, and the corresponding frequencies. The values of these attributes are somehow related to the value of $\epsilon$. For a set of known physical parameters that characterize the well (including its geometry), we can run thousands of simulations, only modifying the unknown parameters (i.e., $\epsilon$ but also the potentially unknown friction weight) between each simulation. From this data set, for which the correct values of $\epsilon$ are known (since these data are simulated), similar to what is done by the human brain (experience), the machine learning algorithm will learn and find the suitable correlations between the previously defined attributes and the unknown parameter $\epsilon$. Once adequately trained, the machine learning algorithm can then be applied to make predictions for new data sets (for which the parameter $\epsilon$ is unknown).

To solve this regression problem and correctly predict the parameter $\epsilon$, we choose to use a neural network algorithm. This kind of Machine Learning algorithm is efficient and easy to implement [? ]. Such a network consists of different connected nodes (that model the neurons of a real brain) called artificial neurons. Each neuron receives different inputs and produces a single output that can be sent to other neurons. The output of each neuron is obtained by applying an activation function to a weighted sum of the inputs. This activation function is usually non-linear. Among the most commonly used activation functions, we can cite the Sigmoid function or the tanh function. More details on how to choose the activation function or the weight can be found in [? ? ]. A neural network can have several layers. The input of the first layers corresponds to the attributes we have chosen (in our case, the attributes that characterize the spectrum of the top-drive force and velocity), while the inputs for the next layers correspond to the neurons' outputs in the previous 
layer. The global output of the neural network is the predicted value of $\epsilon$.

To train the network, we first simulate thousands of test points for which we know the correct output and measure the corresponding top-drive velocity. We generate the spectrum of the topdrive velocity signal for each simulation and choose a set of relevant features (such as the dominant peak, the corresponding frequency) that will constitute our dataset. While training our neural network, it appeared that the relevant attributes for learning are the two dominant gains (and their corresponding frequencies) of the top-drive velocity spectrum. This dataset is divided into two sets: the training set and the testing set. We first construct the network (i.e., we choose the number of neurons, layers, weights) and train it on the training set. We then test its performance on the testing set by computing the mean squared error between the real values of $\epsilon$ and the predicted ones. We repeat the procedure, changing the network's parameters and mixing the training and testing set (cross-validation). Finally, we choose the best network (i.e., the one for which the error is minimum) and train the algorithm with the data available. Our algorithm can then be used on unknown datasets.

Such a neural network algorithm is fast, reliable, and easy to implement. However, the main drawback is that it requires many data (i.e., number of simulations) to train the algorithm. Moreover, changing the physical parameters or the well's geometry implies training the algorithm again with a new set of data. The algorithm may also depend on the system's initial condition and the input (although this is not a real problem since we can always consider a rest state and the same input to estimate $\epsilon$ ). A solution to increase the robustness could be to include these parameters into the neural network at the cost of producing a higher number of training points. Another approach to improving the trained model's generalizability is to use transfer learning concepts [? ? ? ]. The details of the algorithm is summarized in Algorithm 3.

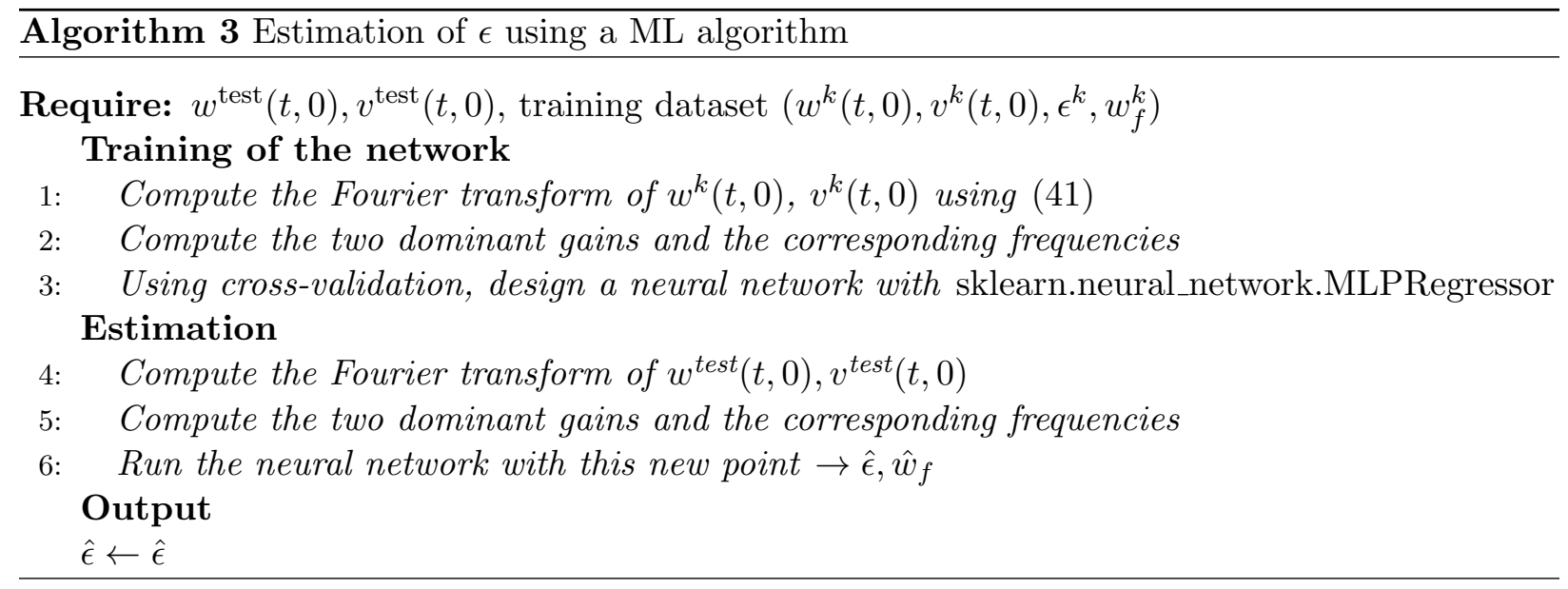

\section{Simulation results}

In this section, we test the performance of our different algorithms against simulated data. First, we evaluate the quality of the weight-on-bit estimations and the bit velocity provided by equations (26) and (27) in the presence of noisy measurements. Then, we will compare the efficiency of our three algorithms in estimating the nature of the rock interacting with the drill-bit.

The axial dynamics simulated adjusting the model of [? ]. The control input $w_{0}$ is chosen as a constant to which is added a sinusoidal function. In the model's numerical implementation, the 


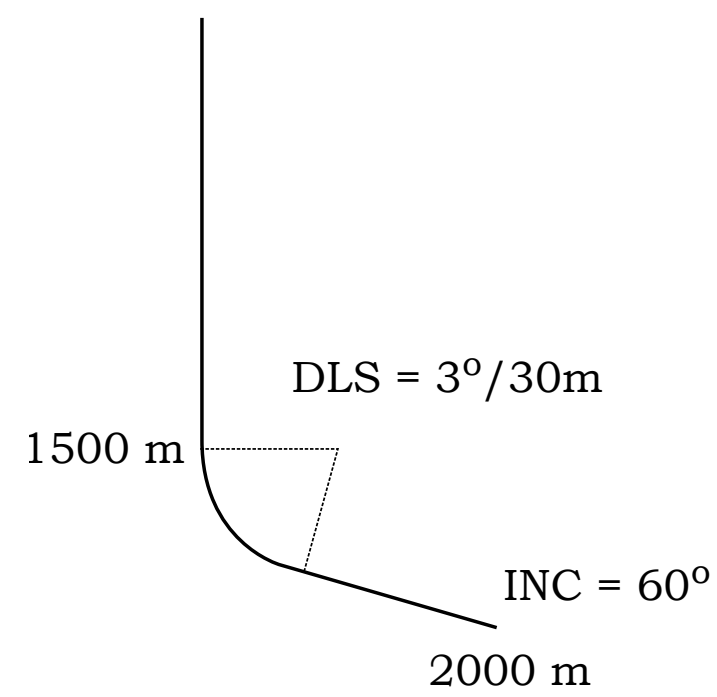

Figure 6: Wellbore survey of the well. The length of the drill-string is $1750 \mathrm{~m}$.

Table 2: Numerical values of the parameters for the drill string model in Figure 6 .

\begin{tabular}{|c|c||c|c|}
\hline Param. & Value & Param. & Value \\
\hline \hline$E^{1}$ & $2 \times 10^{11} \mathrm{~Pa}$ & $\rho^{1}$ & $8000 \mathrm{~kg} / \mathrm{m}^{3}$ \\
$c_{\xi}^{1}$ & $5000 \mathrm{~m} / \mathrm{s}$ & $k_{\xi}^{1}$ & $0.23 / \mathrm{s}$ \\
$A^{1}$ & $3.5 \times 10^{-3} \mathrm{~m}^{2}$ & $L$ & $2000 \mathrm{~m}$ \\
$E^{2}$ & $2.5 \times 10^{11} \mathrm{~Pa}$ & $\rho^{2}$ & $8500 \mathrm{~kg} / \mathrm{m}^{3}$ \\
$c_{\xi}^{2}$ & $5423 \mathrm{~m} / \mathrm{s}$ & $k_{\xi}^{2}$ & $0.3 / \mathrm{s}$ \\
$A^{2}$ & $5 \times 10^{-2} \mathrm{~m}^{2}$ & $x_{1}$ & $1750 \mathrm{~m}$ \\
$M_{b}$ & $30000 \mathrm{~kg}$ & $\omega_{b i t}$ & $5 \mathrm{rad} / \mathrm{s}$ \\
$a$ & $0.1 \mathrm{~m}$ & $\zeta$ & 0.6 \\
$w_{f}$ & $71280 \mathrm{~N}$ & & \\
\hline \multicolumn{4}{|r}{} \\
\hline
\end{tabular}

wave equation is transformed into transport equations discretized using a first-order upwind scheme. This choice is made to ensure numerical robustness and avoid spurious oscillations, as higherorder schemes perform poorly due to the temporal discontinuities introduced by the distributed differential inclusions used to represent the Coulomb friction. To guarantee numerical accuracy, we choose a sufficiently fine spatial grid. This is an amenable approach since the simulation speed is not of critical importance for the present analysis (only the computational efforts of the estimation algorithms matter). In all simulations, a spatial grid of 500 cells is used for the drill string. We choose the time-step such that the Courant-Friedrichs-Lewy (CFL) condition [? ] is satisfied. All the simulations are done using MATLAB except the neural network algorithm that is performed using Python.

\subsection{Estimation of the drill-bit source}

In this section we evaluate the quality of the estimations of the weight-on-bit and of the bit velocity. The drill-bit is interacting with unconsolidated sands (i.e. water saturated) for which $\epsilon=11 \mathrm{Jcm}^{-3}$. The corresponding force-on-bit and bit velocity are denoted $w_{\mathrm{bit}}^{\text {real }}$ and $v_{\mathrm{bit}}^{\text {real }}$ while 
their estimations denoted $w_{\text {bit }}^{\text {est }}$ and $v_{\text {bit }}^{\text {rest }}$. These estimations are obtained using equations (26) and (27). The kernels $K^{*}$ are computed using the method of characteristics and a fixed point algorithm (see [? ] for details). The different integrals are computed using a trapezoidal method with adjustable precision. We consider the case of noisy measurements. We model the noise by a white Gaussian noise that is characterized by its signal-to-noise ratio (SNR). As it is done in [? ], the SNR is defined as

$$
S N R=\frac{a_{r m s}^{2}}{\sigma_{n}^{2}},
$$

where $a_{r m s}$ is the root-mean-square amplitude of the noise-free signal, and $\sigma_{n}^{2}$ is the variance of the noise. Choosing a value for the SNR gives the corresponding amplitude for the white Gaussian noise. Note that the value of the SNR may be different for the velocity and force measurements. We compare the real force-on-bit with our estimation in Figure 7. The comparison between the real axial bit velocity and the estimated one is done in Figure 8 . The SNR is equal to 10 . The estimation is only performed for $\frac{x_{1}}{c_{\xi}^{1}}+\frac{L-x_{1}}{c_{\xi}^{2}}<t<T_{f}-\left(\frac{x_{1}}{c_{\xi}^{1}}+\frac{L-x_{1}}{c_{\xi}^{2}}\right)$, since we cannot compute all the terms present in equations (26) and (27) out of this time interval (we recall that $x_{1}$ corresponds to the length of the pipe-section). To reduce the effect of the noise we use a second order Butterworth low-pass filter on the noisy measurements. The functions $w_{\text {bit }}^{\text {real }}$ and $w_{\text {bit }}^{\text {est }}$ are plotted in Figure 7 . while the functions $v_{\mathrm{bit}}^{\mathrm{est}}$ and $v_{\mathrm{bit}}^{\mathrm{est}}$ are plotted in Figure 8. We notice that despite the presence of noise, the estimations are comparable to the real states. For a space grid of 500 cells, our algorithm needs 90 seconds to compute $v_{\text {bit }}^{\text {est }}$ and $v_{\text {bit }}^{\text {est }}$. This computational time is directly related to the space-step (and consequently to the time step, with the CFL condition). However, most of the computations can be done off-line (computation of the backstepping kernels for instance), and the real computational time is around 20 s to estimate on a 20 s time-window. This makes the current code amenable for real implementation, although in that case complexity of the algorithm should also be considered. Note that the computational burden can be reduced by using a less time-consuming algorithm to compute the different integral terms or by decreasing the space-step (at the cost of a lower accuracy).

As is done in [? ? ] we introduce the quality of the reconstruction metric for the estimated forceon-bit. This will help us to examine the performance of the proposed method. A similar verification is done for the bit velocity. Let us denote $y_{0}$ as true generic signal (in our case $y_{0}=w_{b i t}^{r e a l}$ ) and $y$ its estimation (in our case $y=w_{b i t}^{e s t}$ ). We define the quality of the reconstruction $Q$ as follows

$$
Q=10 \log \frac{\left\|y_{0}\right\|_{2}^{2}}{\left\|y_{0}-y\right\|_{2}^{2}},
$$

where $\|\cdot\|_{2}$ is the $\ell_{2}$ norm. We have plotted in Figure 9 and Figure 10 the variations of $Q_{F}$ and $Q_{v}$ (reconstruction for the downhole force and downhole velocity) for different values of the SNR of top-drive velocity and the top-drive force (the SNR being chosen between 1 and 15). We have also plotted a dashed line that corresponds to the limit value of $Q=10$. We can observe a symmetrical effect between the SNR of top-drive velocity and the SNR of top-drive force. The critical value of $Q=10$ is not reached for low SNR values $(S N R<2)$. In our simulations, we find that when the quality factor is larger than 10, the rock intrinsic energy estimation algorithms result in satisfactory performances. 


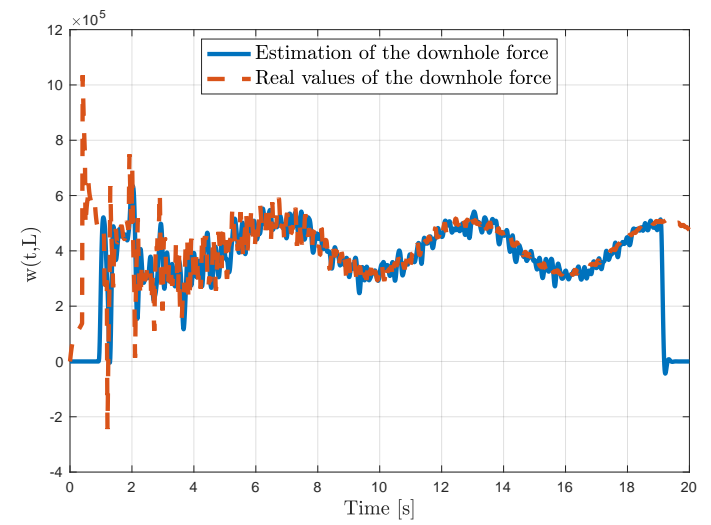

Figure 7: Comparison of the simulated force-onbit and of the estimated one using noisy top-drive measurements in the case of unconsolidated sands $(\mathrm{SNR}=10)$.

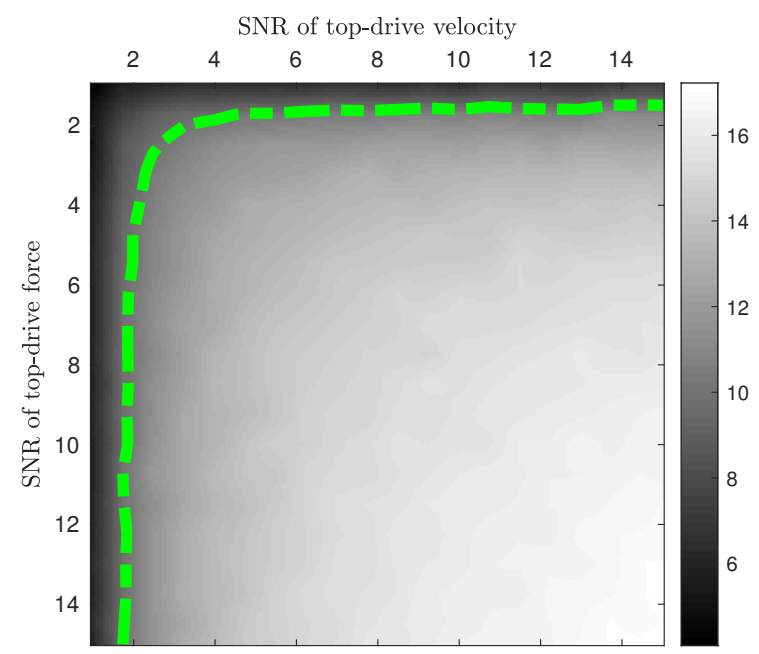

Figure 9: Noise sensitivity analysis of the drill-bit force estimation. We use Monte Carlo simulations with 75 realizations for each noise level pairs and report the average value of the quality of the reconstruction $Q$. The dashed line corresponds to $Q=10$ contour, which is used as the success limit for the drill-bit source estimation.

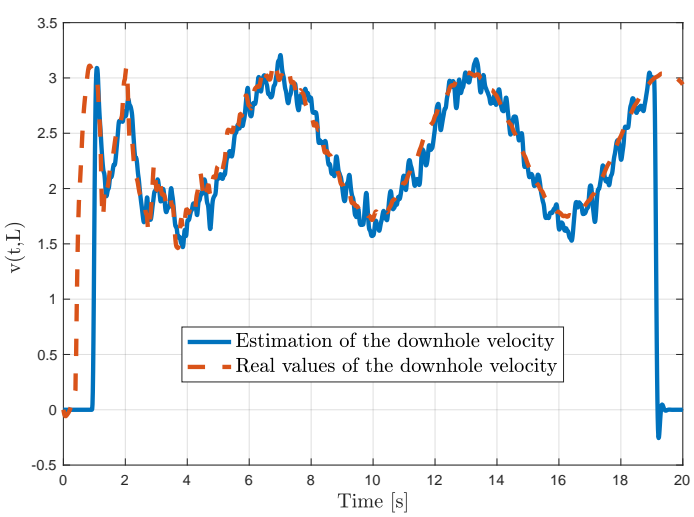

Figure 8: Comparison of the simulated bit velocity and of the estimated one using noisy top-drive measurements in the case of unconsolidated sands $(\mathrm{SNR}=10)$.

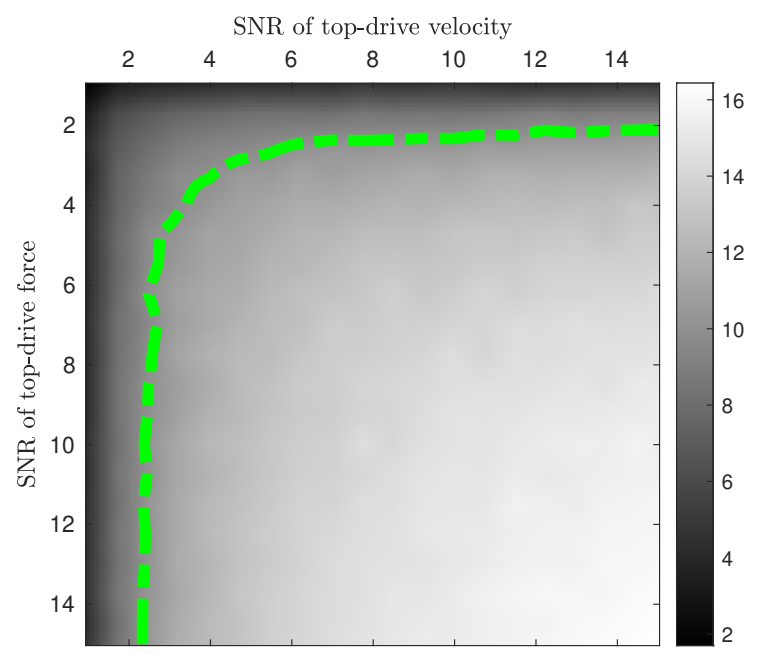

Figure 10: Noise sensitivity analysis of the drill-bit velocity estimation. We use Monte Carlo simulations with 75 realizations for each noise level pairs and report the average value of the quality of the reconstruction $Q$. The dashed line corresponds to $Q=10$ contour, which is used as the success limit for the drill-bit source estimation. 


\subsection{Estimation of the rock interacting with the drill-bit}

We now use our three algorithms to estimate the nature of the rock interacting with the drillbit. The efficiency of the different algorithms are compared for three different types of rocks that have different hardness (i.e., different compressional and shear velocities)

- Unconsolidated sands (i.e. water saturated) for which $V_{p}=2000 \mathrm{~m} / s, V_{s}=1150 \mathrm{~m} / s, \rho_{f}=2$ $\mathrm{g} / \mathrm{cm}^{3}$ and $\epsilon=11 \mathrm{~J} / \mathrm{cm}^{3}$.

- Sedimentary rocks (e.g. sandstone) for which $V_{p}=2710 \mathrm{~m} / \mathrm{s}, V_{s}=1500 \mathrm{~m} / \mathrm{s}, \rho_{f}=2.2$ $\mathrm{g} / \mathrm{cm}^{3}$ and $\epsilon=57 \mathrm{~J} / \mathrm{cm}^{3}$.

- Igneous or metamorphic rocks (e.g. granite) for which $V_{p}=4610 \mathrm{~m} / \mathrm{s} V_{s}=2700 \mathrm{~m} / \mathrm{s}$, $\rho_{f}=2.7 \mathrm{~g} / \mathrm{cm}^{3}$ and $\epsilon=175 \mathrm{~J} / \mathrm{cm}^{3}$.

\subsubsection{Seismic While Drilling Algorithm}

We use our first algorithm (SWD Algorithm) to estimate the rocks' velocity that are interacting with the drill-bit. We consider that 100 seismic sensors are available (50 on each side of the drillstring). The distance between each receiver is equal to 25 meters. Note that this distribution may not be optimal due to the bit's final orientation (which is equal to 60 degrees according to Figure 6). Using the simulated weight-on-bit and the (known) value of $V_{p}$, we compute the radiation patterns for each sensor to which we add noise with SNR equal to 5. Moreover, by using the top-drive force and velocity measurements, which are given by the PDE simulation, we estimate the weight-on-bit, and consequently, it becomes possible to apply Algorithm 1. For the three different rocks, we have performed 75 simulations and compute the corresponding $\epsilon$ using such a procedure. We have given in Table 3 the value of the mean and the standard deviation of our estimations. One can notice that the estimations are almost equal to the real values even in the presence of significant noise. Moreover, the standard deviation remains extremely low. This underlines the robustness of the proposed approach that uses a large number of sensors. For one testing point, Algorithm 1 runs in $0.06 \mathrm{~s}$.

\subsubsection{Direct Estimation Algorithm}

Let us now estimate $\epsilon$ using Algorithm 2. The results are shown in Table 3. We have assumed that the constant $C$ that appears in $(39)$ is not known. One can notice that the estimations are close to the real data (even if, due to the important noise (SNR equal to 5 ), the standard deviation may be important. However, one must keep in mind that such an approach is hugely dependent on the model we have for the downhole boundary condition. The computational time to run Algorithm 2 on one testing point is of $0.01 \mathrm{~s}$.

\subsubsection{Machine Learning Algorithm}

We now design our machine learning algorithm. As explained in Section 5.2 and Algorithm 3 , we first train our algorithm on a set of data made of 10000 simulations for which only the values of $\epsilon$ and $w_{f}$ change between each simulation. An example of a power spectrum of top-drive velocity, for the case $\epsilon=11$, is given in Figure 11. Note that the spectrum has been normalized by its maximum gain and that a saturation has been set at $-20 \mathrm{~dB}$.

To run our neural network, we have used Python's algorithm sklearn.neural_network.MLPRegressor with 50 hidden layers, a BFGS solver for weight optimization, and an adaptive learning rate. Again, the results are shown in Table 3 . One can notice that these predictions are close to the real values 


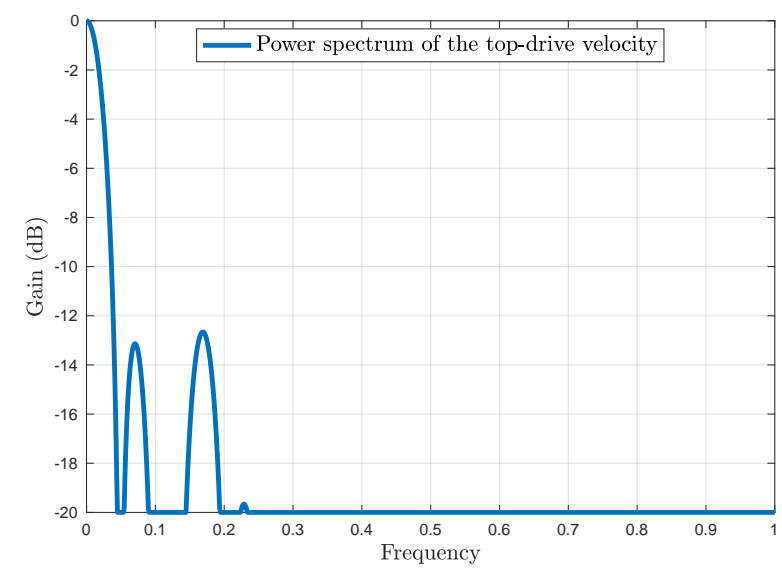

Figure 11: Normalized power spectrum of noise-free top-drive velocity. Saturation is set at $-20 \mathrm{~dB}$.

Table 3: Mean and standard deviation of the intrinsic specific energy of the rock $\epsilon$ using SWD estimation (100 sensors), direct estimation and Machine Learning estimation. 75 simulations and estimations have been performed for each rock. The SNR for all the signals is equal to 5 .

\begin{tabular}{|c|c|c|c|c|c|}
\hline Rock & Real $\epsilon$ & & SWD & Direct Estimation & Machine Learning \\
\hline \hline Unconsolidated & 11 & Mean & 11.1 & 10.2 & 9.7 \\
sands & & Stand. Dev. & 0.4 & 1.1 & 2 \\
\hline Sedimentary & 57 & Mean & 57.2 & 53.5 & 60.9 \\
Rocks & & Stand. Dev. & 0.32 & 2.19 & 3.3 \\
\hline Metamorphic & \multirow{2}{*}{175} & Mean & 175.38 & 186 & 184 \\
Rocks & & Stand. Dev. & 0.5 & 24 & 17.8 \\
\hline
\end{tabular}

(at least for low to medium $\epsilon$ ). The prediction is more accurate for large values of $\epsilon$ (metamorphic rocks) than the one we obtained using the two other algorithms. The computational time to train the network is $140 \mathrm{~s}$ while the time required to run the algorithm on one testing point is $0.01 \mathrm{~s}$.

\subsubsection{Comparison between the different algorithms}

The three different algorithms provide satisfying and reliable estimation for the rock's intrinsic specific energy in the presence of important noises. However, the SWD algorithm presents an impressively high accuracy with a low standard deviation. This is related to its inherent robustness properties due to the use of a large number of sensors. Moreover, the estimation procedure is simple and can be easily adjusted for new constant physical parameters. The second algorithm also gives reliable estimates (at least for low values of $\epsilon$ ). However, one must be aware that this algorithm requires a reliable model for the downhole condition (bit rock-interaction). Finally, even if the Machine Learning algorithm shows satisfactory performance, it requires important datasets to be run, which can be time-consuming. Note that another drawback of our ML procedure is that the available data currently comes from simulations, which implies a model dependency (although it is not as crucial as for the second procedure). However, such a Machine Learning algorithm is easy to implement and fast to run once adequately trained. Moreover, it is not sensitive to the discretization step since it only uses top-drive measurements. 
We now compare the robustness properties of the different algorithms concerning uncertainties on the different physical parameters. More precisely, we address the effects of mismatch between the physical and mathematically modeled drill string dynamics (especially, errors in downhole boundary condition: bit-rock interaction modeling) on the three algorithms' performances. Adding discrepancies to the modeling will introduce errors in estimating the force-on-bit but not the measured hook-load, hook-speed, and SWD measurements. Hence, in the SWD algorithm, an incorrect source will be correlated with the data. In the direct estimation algorithm, the effect of incorrect bit-rock interaction coefficients will be added to a wrong estimation of the force-onbit. Finally, the machine learning algorithm will be trained with the wrong model. The proposed analysis is the first step towards a complete sensitivity analysis (that would be outside the scope of this paper). In what follows, we consider that all the physical parameters used to simulate the drilling system (e.g., inertia, inclination, length, mass) are subject to a random uncertainty (bounded by a maximum percentage $d$ ). We have pictured in Figure 12 the comparison between the simulated force-on bit and the estimated one in the absence of noise but in the presence of a discrepancy of $10 \%$ between the physical parameters used for the simulation and those used for the simulation. Note that for this simulation, all the uncertainties have been set to the maximum bound of $10 \%$. As expected, there is a mismatch between the estimated force and the simulated one (around $15 \%$ ). Note that the phase of the function is barely changed. We now compare our three algorithms' performance in the presence of uncertainties on the different parameters. We consider the case of sedimentary rock for which $\epsilon=57$. We have performed 75 simulations for which there is a discrepancy up to $d \%$ between the parameters used for the estimation (and for the training of the last algorithm) and the real ones. We have given in Table 4 the values of the mean and the standard deviation of our estimations for different maximal bound $d(10 \%, 20 \%$ and $30 \%)$. We have used an SNR of 10 for all the measured signals. All three algorithms provide relevant estimations even in the presence of important uncertainties. The machine learning algorithm seems to be the most accurate but suffers from an important standard deviation. A similar dispersion of the estimations can be observed for the direct estimation algorithm. This is not surprising since these two algorithms are trained on a false model. Conversely, the SWD algorithm gives an accurate estimation with a relatively low standard deviation. We can explain these good performances by the fact that the uncertainties barely affect the phase of the estimated force-on-bit. As the phase is the most important attribute when correlating the estimated force with SWD measurements, this results in better estimation and a lower standard deviation. This emphasizes the robustness of such an approach even if this is done at the cost of an important number of sensors. That said, the SWD method (i.e., Algorithm 1) is an alternating minimization method, which requires proper initial estimation of the formation velocity. In Algorithm 1, we use the time delay between the recorded data near the surface and the calculated force-on-bit (source signature) at the bit location to provide an initial estimation of formation velocity, i.e., $V_{p}^{0}$. This method may not always provide a proper initial estimation of formation velocity. Accordingly, we can take advantage of the direct estimation and machine learning methods in providing $V_{p}^{0}$ necessary in the SWD algorithm. Moreover, in the

SWD algorithm, having access to a proper $V_{p}^{0}$ value will improve the robustness and convergence rate of the SWD algorithm, as well.

\section{Extensions}

The three algorithms we design in this paper provide an efficient and reliable estimation of the rock's nature that is interacting with the drill-bit, enabling a more precise characterization of 


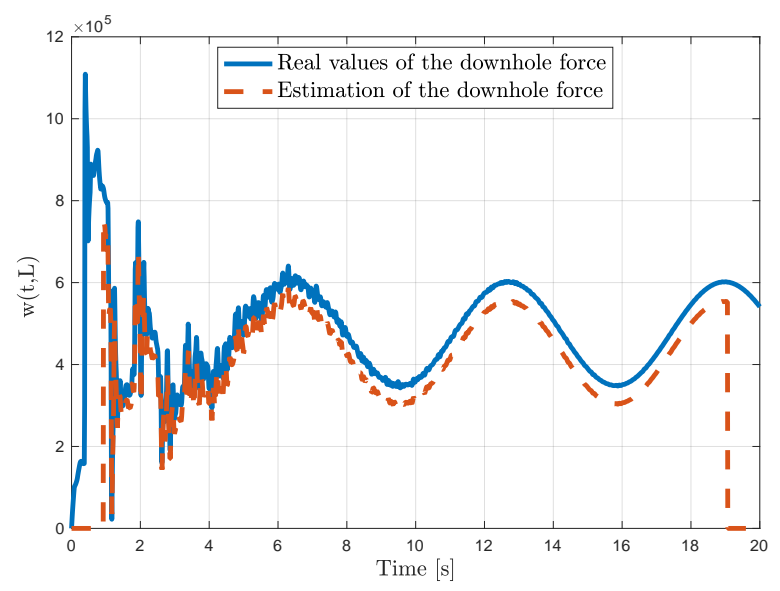

Figure 12: Comparison of the simulated force-on bit and of the estimated one in the presence of a discrepancy of $10 \%$ between the physical parameters used for the simulation and those used for the simulation (no noise)

Table 4: Influence of a parameter mismatch on the estimation of the intrinsic specific energy of a sedimentary rock $\epsilon$ (real value: 57) for each algorithm (SWD estimation (100 sensors), direct estimation and Machine Learning estimation). 75 simulations and estimations have been performed for each level of disturbance. The SNR for all the signals is equal to 5 .

\begin{tabular}{|c|c|c|c|c|}
\hline Disturbance & & SWD & Direct Estimation & Machine Learning \\
\hline \hline $10 \%$ & Mean & 54.7 & 51.6 & 57.5 \\
& Stand. Dev. & 2.47 & 6 & 7.6 \\
\hline $20 \%$ & Mean & 53.7 & 50 & 57.5 \\
& Stand. Dev. & 10.7 & 12.5 & 17 \\
\hline $30 \%$ & Mean & 48.9 & 46.1 & 58.4 \\
& Stand. Dev. & 11 & 18 & 21 \\
\hline
\end{tabular}

the subsurface. However, the proposed axial dynamics given by equations (3)-(4) neglect several aspects that are crucial to envision real implementation. First, we have neglected the Coulomb friction between the drill string and the borehole. We have then neglected the torsional dynamics of the drilling device by assuming that the bit angular velocity was perfectly known. The main reason behind this choice of an (over)-simplistic model was to facilitate the design of our different procedures. This section shows how our algorithms can be extended to these more sophisticated (and realistic) models.

\subsection{Non-linear Coulomb friction terms}

The proposed (simplified) axial dynamics given by equations (3)-(4) neglect the Coulomb friction between the drill string and the borehole (also known as side force). It has been shown in [? ], that for torsional oscillations, this Coulomb friction term may have a non-negligible impact on the dynamics. More precisely, it has a velocity weakening effect that may generate stick-slip oscillations. As far as we know, the side force effect on the axial motion of the drill-string has not been studied in the literature. However, it seems reasonable to assume that this force can be expressed using a similar model as the one used for torsional oscillations, namely a differential 
inclusion. More precisely, inspired by [? ] we add the term $\mathcal{F}(t, x)$ to equation (4) that is a differential inclusion that represents the Coulomb friction between the drill-string and the borehole. The model we propose uses the following inclusion

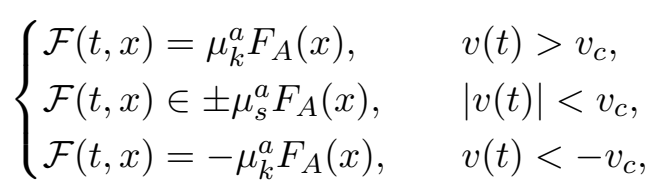

where $\mu_{s}^{a}$ is the static friction coefficient (i.e. the friction between two or more solid objects that are not moving relative to each other) and $\mu_{k}^{a}$ kinetic friction coefficient (also known as dynamic friction or sliding friction, which occurs when two objects are moving relative to each other and rub together), $v_{c}$ is the threshold on the axial velocity where the Coulomb friction transits from static to dynamic. The function $F_{A}$ is the normalized axial force acting between the drill-string and the borehole wall. The function $\mathcal{F}(t, x) \in \pm \mu_{s}^{a} F_{A}(x)$ denotes the inclusion where

$$
\mathcal{F}(t, x)=-\frac{1}{A \rho} \frac{\partial}{\partial x} w(t, x)-k_{\xi} v(t, x) \in\left[-\mu_{s}^{a} F_{A}(x), \mu_{s}^{a} F_{A}(x)\right],
$$

and takes the boundary values $\pm \mu_{s}^{a} F_{A}(x)$ if this relationship does not hold. Let us assume that the coefficients $\mu_{s}^{a}, \mu_{k}^{a}$ and $v_{c}$ are known as well as the function $F_{A}$. How to estimate such coefficients is outside the scope of this paper, but when the bit is off-bottom, adaptive observers can be designed following the procedure detailed in [? ]. When all the points of the drill-string reach the kinematic mode, then the function $\mathcal{F}(t, x)$ does not (directly) depend on time anymore since we have $\mathcal{F}(t, x)= \pm \mu_{k}^{a} F_{A}(x)$. Thus, this term can be added to the function $h$ in (4) and the previous computations can be still be applied to compute the downhole force and velocity as functions of past and future values of the top-drive states. These estimations only hold if we have $|v|>v_{c}$ for all drill-string points. Thus, we need to apply our formula to all points (in practice, to a sufficiently large number of points) of the drill-string to verify that the condition is not violated. Among all the estimations of the drill-bit source signature, only the ones for which this condition is fulfilled can be used to estimate the rock's nature if using Algorithms 1 and 2. Note that using adequate control laws may enforce the system to remain in a steady-state for which this condition is always verified. Regarding the machine learning algorithm, it can straightforwardly be adjusted to deal with this Coulomb friction term. Moreover, by slightly modifying the algorithm, it is also possible to estimate the friction coefficients $\mu_{s}^{a}$ and $\mu_{k}^{a}$. We compare the real force-on-bit with our estimation in Figure 13. The comparison between the real axial bit velocity and the estimated one is made in Figure 13. The different signals are noise-free. The Coulomb friction parameters are chosen as follows:

$$
\mu_{s}^{a}=0.45, \mu_{k}^{a}=0.28, v_{c}=0.25 .
$$

They are assumed to remain the same for the pipe and the collar. One can notice that the downhole force and velocity estimations remain extremely satisfying except for some time intervals for which the velocity is lower than $v_{c}$. This is consistent with our analysis since, in that case, the system has not broken the static friction limit.

\subsection{Torsional motion of the drill-string}

This paper focused on the drill-bit's axial motion and assumed that the bit angular velocity $\omega_{b i t}$ was a known positive function. However, the proposed axial dynamics have to be coupled with 


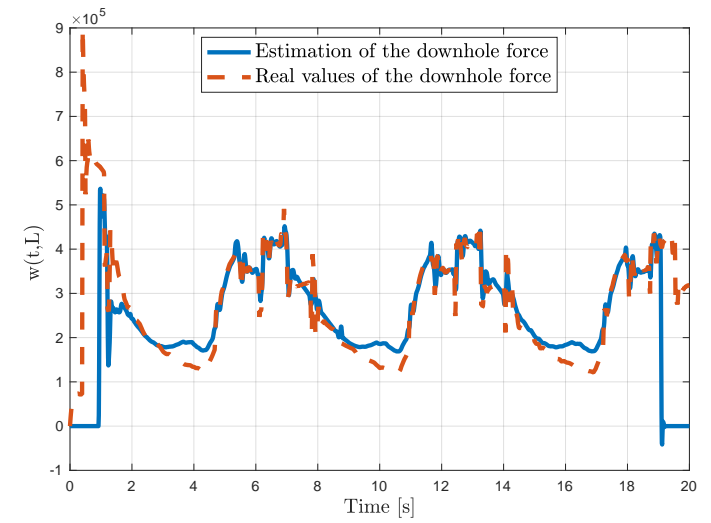

Figure 13: Comparison of the simulated force-onbit and of the estimated one in presence of Coulomb friction terms (no noise).

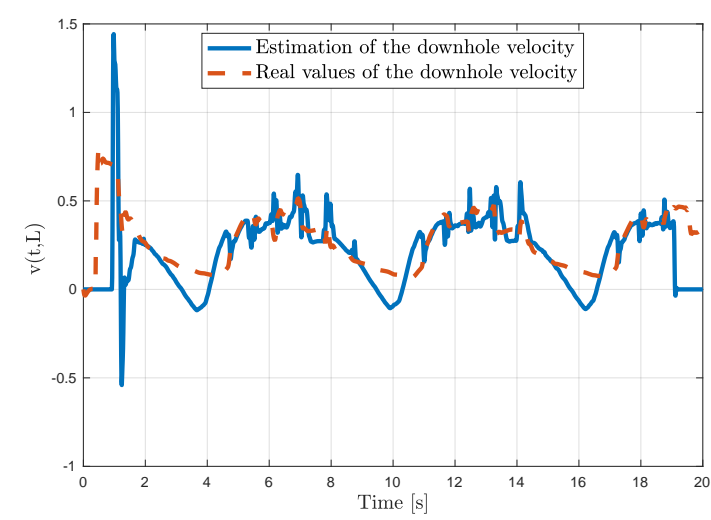

Figure 14: Comparison of the simulated bit velocity and of the estimated one in presence of Coulomb friction terms (no noise).

a torsional model to obtain an axial-torsional drill-string model. Such a torsional model has been proposed in [? ] in the case of an off-bottom bit. The equations have the same structure as (3)-(4), including a non-linear Coulomb friction term similar to (44). Nevertheless, for non-vertical wells, in the presence of angular and axial movements, the Coulomb friction term couples the angular and axial dynamics, and equation (44) has to be modified accordingly. Regarding the downhole boundary conditions (equation (9) and the analogous equation for torque as given in [? ]), as suggested in [? ], the term $\frac{1}{\omega_{\text {bit }}}$ has to replaced by an inclusion ( $g$ non-linearity) to deal with small axial and angular velocities. For torsional oscillations, such a term can be the source of stick-slip oscillations. The derivation of a complete and validated axial-torsional model is out of the scope of this paper. However, even if the downhole boundary conditions become more complex, adjusting the approach presented in Section 4, it becomes possible (eventually including the new Coulomb side forces) to express the bit torque and angular velocity as delayed and future values of the topdrive states. Indeed, our approach does not require the use of the downhole boundary condition. Consequently, our first algorithm can still be applied. However, the second algorithm still requires a good model for the downhole boundary condition. Finally, the machine learning procedure can still be implemented by including the top-drive torque and angular velocity spectrum.

\section{Concluding remarks}

We have developed three different algorithms for a near-real-time estimation of the rock's intrinsic specific energy interacting with the drill-bit. We have considered a multi-sectional directional well. The algorithms only use surface measurements and do not depend on any explicit knowledge about the subsurface properties. The algorithms were based on seismic-while-drilling, direct parameter estimation, and machine learning. They provided an efficient and reliable framework for estimating the nature of rocks interacting with the drill-bit, enabling a subsurface's practical characterization. We have discussed the advantages and drawbacks of each approach. Among our three algorithms, the model-based estimation algorithm (Algorithm 2) provides accurate estimations. However, this algorithm requires a reliable model for the downhole condition and, in that sense, is extremely model-dependent. It is easy to implement and present satisfying robust- 
ness properties regarding the SWD procedure due to the available multiple seismic sensors. The Machine Learning algorithm is also easy to implement and fast to run once properly trained but may not be robust to changes in the system parameters (as a new long training phase would be necessary). We have shown that the proposed methodologies can be applied to drilling systems in the presence of non-linear Coulomb friction terms and coupled axial-torsional oscillations of the drill string. Theoretically, the proposed estimations can be coupled with existing observers and controllers to improve the drilling performances by reducing non-productive time, increasing the $\mathrm{ROP}$, and reducing the effect of undesired torsional oscillations. This is an important step towards a subsurface-aware drilling system. Even though the proposed approach is a necessary and significant step in incorporating formation information and sensing in drill string dynamics estimation, our future interest entails adding more complexities into the modeling system (by combining torsional and axial dynamics), generalizing the machine learning mapping operator, designing formationaware observers for optimal controlling of drill string dynamics, and validating the algorithms in field trail.

\section{Appendix}

\section{Derivation of the delayed equation (26)}

In this appendix, we detail the computations to obtain equation (26). We follow the steps given in [? ], the only differences being that we are between $x_{i}$ and $x_{i+1}$ (instead of 0 and L) and the presence of the functions $h_{3}$ and $h_{4}$ (since the well is not vertical). The transformation (20)-(21) being a Volterra transformation, it is invertible [? ]. The inverse transformation is defined by

$$
\begin{aligned}
& u^{i}(t, x)=\alpha^{i}(t, x)+\int_{x_{i}}^{x}\left(L_{i}^{\alpha \alpha}(x, y) \alpha^{i}(t, y)+L_{i}^{\alpha \beta}(x, y) \beta^{i}(t, y)\right) d y, \\
& z^{i}(t, x)=\beta^{i}(t, x)+\int_{0}^{x_{i}}\left(L_{i}^{\beta \alpha}(x, y) \alpha^{i}(t, y)+L_{i}^{\beta \beta}(x, y) \beta^{i}(t, y)\right) d y,
\end{aligned}
$$

where the kernels $L^{i}$ can be expressed as functions of the kernels $K^{i}$ (see [? ? ]). Injecting (24) and (25) into (46), using Fubini's theorem and simple changes of variables, we obtain (see [? ] for details)

$$
u^{i}\left(t, x_{i+1}\right)=\alpha^{i}\left(t-\frac{\Delta_{i}}{c_{\xi}^{i}}, x_{i}\right)+\int_{-\frac{\Delta_{i}^{i}}{c_{\xi}^{i}}}^{\frac{\Delta_{i}}{c_{\xi}^{i}}} c_{\xi}^{i}\left(f_{1}\right)_{i}^{u}(s) \alpha^{i}\left(t-s, x_{i}\right)+c_{\xi}^{i}\left(f_{2}\right)_{i}^{u}(s) \beta^{i}\left(t-s, x_{i}\right) d s+H_{u}^{i}
$$

where $\left(f_{1}\right)_{i}^{u}$ and $\left(f_{2}\right)_{i}^{u}$ are $L^{\infty}$ functions defined on the interval $\left[-\frac{\Delta_{i}}{c_{\xi}}, \frac{\Delta_{i}}{c_{\xi}}\right]$ by

$$
\begin{aligned}
\left(f_{1}\right)_{i}^{u}(s)= & \mathbb{1}_{\left[0, \frac{\Delta_{i}}{\left.c_{\xi}^{i}\right]}\right.}(s) L_{i}^{\alpha \alpha}\left(x_{i+1}, x_{i}+c_{\xi}^{i} s\right)-\mathbb{1}_{\left[-\frac{\Delta_{i}}{c_{\xi}}, 0\right]}(s) \int_{-c_{\xi}^{i} s+x_{i}}^{x_{i+1}} L_{i}^{\alpha \beta}\left(x_{i+1}, y\right) K_{i}^{z u}\left(y+c_{\xi}^{i} s, x_{i}\right) d y, \\
\left(f_{2}\right)_{i}^{u}(s)= & \mathbb{1}_{\left[-\frac{\Delta_{i}}{\left.c_{\xi}^{i}, 0\right]}\right.}(s)\left(L_{i}^{\alpha \beta}\left(x_{i+1}, x_{i}-c_{\xi}^{i} s\right)\right)-\mathbb{1}_{\left[0, \frac{\Delta_{i}}{\left.c_{\xi}^{i}\right]}\right.}(s)\left(K_{i}^{u z}\left(x_{i+1}-c_{\xi}^{i} s, x_{i}\right)\right. \\
& \left.+\int_{c_{\xi}^{i} s+x_{i}}^{x_{i+1}} L_{i}^{\alpha \alpha}\left(x_{i+1}, y\right) K_{i}^{u z}\left(y-c_{\xi}^{i} s, x_{i}\right) d y\right)
\end{aligned}
$$


and where $H_{u}^{i}$ is defined by

$$
\begin{aligned}
H_{u}^{i}= & \int_{0}^{\frac{\Delta_{i}}{c_{\xi}^{i}}} h_{3}^{i}\left(x_{i+1}-c_{\xi}^{i} y\right) d y+\int_{x_{i}}^{x_{i+1}} \int_{0}^{\frac{y-x_{i}}{c_{\xi}^{i}}}\left(L_{i}^{\alpha \alpha}\left(x_{i+1}, y\right) h_{3}^{i}\left(y-c_{\xi}^{i} \nu\right)\right. \\
& \left.-L_{i}^{\alpha \beta}\left(x_{i+1}, y\right) h_{4}^{i}\left(y-c_{\xi}^{i} \nu\right)\right) d \nu d y .
\end{aligned}
$$

Note that the function $H_{u}^{i}$ was not present in [? ] since the well was considered as vertical which yields $h \equiv 0$. Similarly, the state $z^{i}\left(t, x_{i+1}\right)$ verifies

$$
z^{i}\left(t, x_{x+1}\right)=\beta^{i}\left(t+\frac{\Delta_{i}}{c_{\xi}^{i}}, x_{i}\right)+\int_{-\frac{\Delta_{i}}{c_{\xi}^{i}}}^{\frac{\Delta_{i}}{c_{\xi}^{i}}} c_{\xi}^{i}\left(f_{1}\right)_{i}^{z}(s) \alpha_{i}\left(t-s, x_{i}\right)+c_{\xi}^{i}\left(f_{2}\right)_{i}^{z}(s) \beta^{i}\left(t-s, x_{i}\right) d s+H_{z}^{i}
$$

where $\left(f_{1}\right)_{i}^{z}$ and $\left(f_{2}\right)_{i}^{z}$ are $L^{\infty}$ functions defined on the interval $\left[-\frac{\Delta_{i}}{c_{\xi}}, \frac{\Delta_{i}}{c_{\xi}}\right]$ by

$$
\begin{aligned}
\left(f_{1}\right)_{i}^{z}(s)= & \mathbb{1}_{\left[0, \frac{\Delta_{i}}{\left.c_{\xi}^{i}\right]}\right.}(s)\left(L_{i}^{\beta \alpha}\left(x_{i+1}, x_{i}+c_{\xi}^{i} s\right)\right)-\mathbb{1}_{\left[-\frac{\Delta_{i}}{c_{\xi}}, 0\right]}(s)\left(\int_{-c_{\xi}^{i} s+x_{i}}^{x_{i+1}} L_{i}^{\beta \beta}\left(x_{i+1}, y\right) \cdot K_{i}^{z u}\left(y+c_{\xi}^{i} s, x_{i}\right) d y\right. \\
& \left.+K_{i}^{z u}\left(x_{i+1}+c_{\xi} s, 0\right)\right), \\
\left(f_{2}\right)_{i}^{z}(s)= & \mathbb{1}_{\left[-\frac{\Delta_{i}}{\left.c_{\xi}^{i}, 0\right]}\right.}(s)\left(L_{i}^{\beta \beta}\left(x_{i+1}, x_{i}-c_{\xi}^{i} s\right)\right)-\mathbb{1}_{\left[0, \frac{\Delta_{i}}{c_{\xi}^{i}}\right]}(s)\left(\int_{c_{\xi}^{i} s+x_{i}}^{x_{i+1}} L_{i}^{\beta \alpha}\left(x_{i+1}, y\right) K_{i}^{u z}\left(y-c_{\xi}^{i} s, x_{i}\right) d y\right),
\end{aligned}
$$

and where $H_{z}^{i}$ is defined by

$$
\begin{aligned}
H_{z}^{i}= & -\int_{0}^{\frac{\Delta_{i}}{c_{\xi}^{i}}} h_{4}^{i}\left(x_{i+1}-c_{\xi}^{i} y\right) d y+\int_{x_{i}}^{x_{i+1}} \int_{0}^{\frac{y-x_{i}}{c_{\xi}^{i}}}\left(L_{i}^{\beta \alpha}\left(x_{i+1}, y\right) h_{3}^{i}\left(y-c_{\xi}^{i} \nu\right)\right. \\
& \left.-L_{i}^{\beta \beta}\left(x_{i+1}, y\right) h_{4}^{i}\left(y-c_{\xi}^{i} \nu\right)\right) d \nu d y .
\end{aligned}
$$

Since we have $\alpha^{i}\left(t, x_{i}\right)=u^{i}\left(t, x_{i}\right)$ and $\beta^{i}\left(t, x_{i}\right)=z^{i}\left(t, x_{i}\right)$ and since

$$
\begin{aligned}
& w\left(t, x_{i+1}\right)=\frac{E^{i} A^{i}}{2 c_{\xi}^{i}}\left(\mathrm{e}^{-\frac{k_{\xi}^{i} x_{i+1}}{2 c_{\xi}^{i}}} u^{i}\left(t, x_{i+1}\right)-\mathrm{e}^{\frac{k_{\xi}^{i} x_{i+1}}{2 c_{\xi}^{i}}} z^{i}\left(t, x_{i+1}\right)\right), \\
& v\left(t, x_{i+1}\right)=\frac{1}{2}\left(\mathrm{e}^{-\frac{k_{\xi}^{i} x_{i+1}}{2 c_{\xi}^{i}}} u^{i}\left(t, x_{i+1}\right)+\mathrm{e}^{\frac{k_{\xi}^{i} x_{i+1}}{2 c_{\xi}^{i}}} z^{i}\left(t, x_{i+1}\right)\right),
\end{aligned}
$$

injecting (48) and (50) into these equations, we immediately obtain (26) and (27) where we have

$$
\begin{aligned}
f_{u}^{i}(s) & =\frac{E^{i} A^{i}}{2}\left(\mathrm{e}^{-\frac{k_{\xi}^{i} x_{i+1}}{2 c_{\xi}}}\left(f_{1}\right)_{i}^{u}(s)-\mathrm{e}^{\frac{k_{\xi}^{i} x_{i+1}}{2 c_{\xi}^{i}}}\left(f_{1}\right)_{i}^{z}(s)\right), \\
f_{z}^{i}(s) & =\frac{E^{i} A^{i}}{2}\left(\mathrm{e}^{-\frac{k_{\xi}^{i} x_{i+1}}{2 c_{\xi}^{i}}}\left(f_{2}\right)_{i}^{u}(s)-\mathrm{e}^{\frac{k_{\xi}^{i} x_{i+1}}{2 c_{\xi}^{i}}}\left(f_{2}\right)_{i}^{z}(s)\right), \\
g_{u}^{i}(s) & =\frac{c_{\xi}^{i}}{2}\left(\mathrm{e}^{-\frac{k_{\xi}^{i} x_{i+1}}{2 c_{\xi}}}\left(f_{1}\right)_{i}^{u}(s)+\mathrm{e}^{\frac{k_{\xi}^{i} x_{i+1}}{2 c_{\xi}^{i}}}\left(f_{1}\right)_{i}^{z}(s)\right), \\
g_{z}^{i}(s) & =\frac{c_{\xi}^{i}}{2}\left(\mathrm{e}^{-\frac{k_{\xi}^{i} x_{i+1}}{2 c_{\xi}^{i}}}\left(f_{2}\right)_{i}^{u}(s)+\mathrm{e}^{\frac{k_{\xi}^{i} x_{i+1}}{2 c_{\xi}^{i}}}\left(f_{2}\right)_{i}^{z}(s)\right) .
\end{aligned}
$$




\section{Acknowledgments}

This work was partially supported by the University of Calgary's Canada First Research Excellence Fund Program, the Global Research Initiative in Sustainable Low Carbon Unconventional Resources.

\section{References}

[] U. J. F. Aarsnes, F. Di Meglio, R. J. Shor, Avoiding stick slip vibrations in drilling through startup trajectory design, Journal of Process Control 70 (2018) 24-35. URL: https://linkinghub.elsevier.com/retrieve/pii/ S0959152418301859 doi $10.1016 / \mathrm{j} \cdot$ jprocont.2018.07.019

[] U. J. F. Aarsnes, F. di Meglio, R. Shor, Benchmarking of industrial stick-slip mitigation controllers, IFACPapersOnLine 51 (2018) 233-238.

[] V. A. Dunayevsky, F. Abbassian, Application of Stability Approach to Bit Dynamics, SPE Drilling \& Completion 13 (1998) 22-25. URL: https://www onepetro.org/journal-paper/SPE-30478-PA doi 10.2118/30478-PA

[] J. D. Jansen, Nonlinear dynamics of oilwell drillstrings, Ph.D. thesis, Delft University of Technology, 1993. URL: http://repository.tudelft.nl/assets/uuid:d205b287-bcdd-4b49-b12a-963d9b906dea/ 3me\{_\}jansen\{_\}19930616.PDF

[] B. Saldivar, S. Mondié, J.-J. Loiseau, V. Rasvan, Stick-slip oscillations in oillwell drilstrings: Distributed parameter and neutral type retarded model approaches, IFAC Proceedings Volumes (IFAC-PapersOnline) 18 (2011) 284-289. doi 10.3182/20110828-6-IT-1002.00084.

[] B. Saldivar, I. Boussaada, H. Mounier, S.-I. Niculescu, Analysis and control of oilwell drilling vibrations, in: A Time-Delay Systems Approach, ser. Advances in Industrial Control, Springer, 2015.

[] P. C. Kriesels, W. . J. G. Keultjes, P. Dumont, I. Huneidi, O. Owoeye, R. A. Hartmann, et al., Cost savings through an integrated approach to drillstring vibration control, in: SPE/IADC Middle East Drilling Technology Conference, Society of Petroleum Engineers, 1999.

[] U. J. Aarsnes, R. J. Shor, Torsional vibrations with bit off bottom: Modeling, characterization and field data validation, Journal of Petroleum Science and Engineering 163 (2018) 712-721. URL: http://linkinghub. elsevier.com/retrieve/pii/S0920410517309075 doi 10.1016/j.petrol.2017.11.024

[] R. I. Leine, D. H. van Campen, W. J. G. Keultjes, Stick-slip Whirl Interaction in Drillstring Dynamics, Journal of Vibration and Acoustics 124 (2002) 209. URL: http://vibrationacoustics .asmedigitalcollection.asme. org/article.aspx?articleid=1470408 doi $10.1115 / 1.1452745$

[] K. Nandakumar, M. Wiercigroch, Stability analysis of a state dependent delayed, coupled two DOF model of drill-string vibration, Journal of Sound and Vibration 332 (2013) 2575-2592. URL: http://linkinghub. elsevier.com/retrieve/pii/S0022460X1200987X doi 10.1016/j·jsv.2012.12.020

[] J. F. Brett, The Genesis of Bit-Induced Torsional Drillstring Vibrations, SPE Drilling Engineering 7 (1992) 168-174. URL: http://www.onepetro.org/mslib/servlet/onepetropreview?id=00021943\{\&\}soc= SPEhttp://www.onepetro.org/doi/10.2118/21943-PA doi:10.2118/21943-PA.

[] M. Kapitaniak, V. V. Hamaneh, J. P. Chávez, K. Nandakumar, M. Wiercigroch, Unveiling complexity of drill-string vibrations: Experiments and modelling, International Journal of Mechanical Sciences 101 (2015) 324-337.

[] J. F. Brett, A. D. Beckett, C. A. Holt, D. L. Smith, Uses and Limitations of Drillstring Tension and Torque Models for Monitoring Hole Conditions, SPE Drilling Engineering 4 (1989) 223-229. URL: http: //www.onepetro.org/doi/10.2118/16664-PA doi 10.2118/16664-PA.

[] G. W. Halsey, A. Kyllingstad, T. V. Aarrestad, D. Lysne, Drillstring Vibrations: Comparison Between Theory and Experiments on a Full-Scale Research Drilling Rig, in: SPE/IADC Drilling Conference, IADC/SPE 14760, Society of Petroleum Engineers, 1986, pp. 311-321. URL: http://www.onepetro.org/doi/10.2118/14760-MS. doi $10.2118 / 14760-\mathrm{MS}$

[] D. Zhao, S. Hovda, S. Sangesland, Abnormal Down Hole Pressure Variation by Axial Stick-Slip of Drillstring, Journal of Petroleum Science and Engineering 145 (2016) 194-204. URL: http://dx.doi.org/10.1016/ $610 \quad j \cdot$ petrol.2016.04.004 doi $10.1016 / j \cdot$ petrol.2016.04.004

[] F. Di Meglio, U. J. F. Aarsnes, A distributed parameter systems view of control problems in drilling, IFACPapersOnLine 48 (2015) 272-278.

[] B. Saldivar, S. Mondié, S.-I. Niculescu, H. Mounier, I. Boussaada, A control oriented guided tour in oilwell drilling vibration modeling, Annual Reviews in Control 42 (2016) 100-113. 
[] U. J. F. Aarsnes, O. M. Aamo, Linear stability analysis of self-excited vibrations in drilling using an infinite dimensional model, Journal of Sound and Vibration 360 (2016) 239-259. URL: http://linkinghub.elsevier. com/retrieve/pii/S0022460X15007385 doi 10.1016/j.jsv.2015.09.017.

[] U. J. F. Aarsnes, N. van de Wouw, Axial and torsional self-excited vibrations of a distributed drill-string, Journal of Sound and Vibration 444 (2019) 127-151.

[] C. Germay, V. Denoël, E. Detournay, Multiple mode analysis of the self-excited vibrations of rotary drilling systems, Journal of Sound and Vibration 325 (2009) 362-381. URL: http://linkinghub.elsevier.com/retrieve/ pii/S0022460X09002478 doi $10.1016 / j \cdot j s v .2009 .03 .017$

[] U. J. F. Aarsnes, R. J. Shor, Torsional vibrations with bit off bottom: Modeling, characterization and field data validation, Journal of Petroleum Science and Engineering 163 (2018) 712-721.

[] I. Boussaada, H. Mounier, S.-I. Niculescu, A. Cela, Analysis of drilling vibrations: A time-delay system approach, in: 2012 20th Mediterranean Conference on Control \& Automation (MED), IEEE, 2012, pp. 610-614.

[] T. Richard, C. Germay, E. Detournay, Self-excited stick-slip oscillations of drill bits, Comptes Rendus Mécanique 332 (2004) 619-626. URL: http://linkinghub.elsevier.com/retrieve/pii/S1631072104001135. doi $10.1016 /$ j.crme.2004.01.016

[] T. Richard, C. Germay, E. Detournay, A simplified model to explore the root cause of stick-slip vibrations in drilling systems with drag bits, Journal of Sound and Vibration 305 (2007) 432-456. URL: http://linkinghub. elsevier.com/retrieve/pii/S0022460X07002908 doi 10.1016/j·jsv.2007.04.015.

[] L. Franca, Drilling action of roller-cone bits: modeling and experimental validation, Journal of Energy Resources Technology 132 (2010).

[] Z. Zhao, X. He, Z. Ren, G. Wen, Boundary adaptive robust control of a flexible riser system with input nonlinearities, IEEE Transactions on Systems, Man, and Cybernetics: Systems 49 (2018) 1971-1980.

[] Y. Liu, Y. Fu, W. He, Q. Hui, Modeling and observer-based vibration control of a flexible spacecraft with external disturbances, IEEE Transactions on Industrial Electronics 66 (2018) 8648-8658.

[] X. He, W. He, J. Shi, C. Sun, Boundary vibration control of variable length crane systems in two-dimensional space with output constraints, IEEE/ASME Transactions on Mechatronics 22 (2017) 1952-1962.

[] Y. Liu, F. Guo, X. He, Q. Hui, Boundary control for an axially moving system with input restriction based on disturbance observers, IEEE Transactions on Systems, Man, and Cybernetics: Systems 49 (2018) 2242-2253.

[] M. Liao, J. Ing, M. Sayah, M. Wiercigroch, Dynamic method of stiffness identification in impacting systems for percussive drilling applications, Mechanical Systems and Signal Processing 80 (2016) 224-244.

[] M. Liao, M. Wiercigroch, M. Sayah, J. Ing, Experimental verification of the percussive drilling model, Mechanical Systems and Signal Processing 146 (2021) 107067.

[] R. J. Shor, M. W. Dykstra, O. J. Hoffmann, M. Coming, For better or worse: applications of the transfer matrix approach for analyzing axial and torsional vibration, in: SPE/IADC Drilling Conference and Exhibition, Society of Petroleum Engineers, 2015.

[] Z. Zhao, X. He, C.-K. Ahn, Boundary disturbance observer-based control of a vibrating single-link flexible manipulator, IEEE Transactions on Systems, Man, and Cybernetics: Systems (2019).

[] Z. Zhao, C.-K. Ahn, H.-X. Li, Boundary antidisturbance control of a spatially nonlinear flexible string system, IEEE Transactions on Industrial Electronics 67 (2019) 4846-4856.

[] J. Auriol, N. Kazemi, R. J. Shor, K. A. Innanen, I. D. Gates, A sensing and computational framework for estimating the seismic velocities of rocks interacting with the drill bit, IEEE Transactions on Geoscience and Remote Sensing 58 (2019) 3178-3189.

[] F. Poletto, F. Miranda, Seismic while drilling: Fundamentals of drill-bit seismic for exploration, volume 35, Elsevier, 2004.

[] N. Kazemi, R. Shor, K. Innanen, Illumination compensation with seismic-while-drilling plus surface seismic imaging, in: 80th EAGE Conference and Exhibition 2018, volume 2018, European Association of Geoscientists \& Engineers, 2018, pp. 1-5.

[] S. Nejadi, N. Kazemi, J. A. Curkan, J. Auriol, P. R. Durkin, S. M. Hubbard, K. A. Innanen, R. J. Shor, I. D. Gates, et al., Look ahead of the bit while drilling: Potential impacts and challenges of acoustic seismic while drilling in the mcmurray formation, SPE Journal (2020).

[] N. Kazemi, J. Auriol, K. Innanen, R. Shor, I. Gates, Successive full-waveform inversion of surface seismic and seismic-while-drilling datasets without low frequencies, in: 82nd EAGE Annual Conference \& Exhibition, volume 2020, European Association of Geoscientists \& Engineers, 2020, pp. 1-5.

[] J. V. Beck, K. J. Arnold, Parameter estimation in engineering and science, James Beck, 1977.

[] S. Chen, S. A. Billings, P. M. Grant, Non-linear system identification using neural networks, International journal of control 51 (1990) 1191-1214. 
[] I. Goodfellow, Y. Bengio, A. Courville, Deep learning, MIT press, 2016.

[] Y. Liu, W. Zhan, M. Xing, Y. Wu, R. Xu, X. Wu, Boundary control of a rotating and length-varying flexible robotic manipulator system, IEEE Transactions on Systems, Man, and Cybernetics: Systems (2020).

[] J.-B. Kim, S.-J. Lee, Y.-P. Park, Stable and efficient drilling process by active control of the thrust force, Mechanical Systems and Signal Processing 8 (1994) 585-595.

[] Y. Liu, X. Chen, Y. Mei, Y. Wu, Observer-based boundary control for an asymmetric output-constrained flexible robotic manipulator, SCIENCE CHINA Information Sciences (2020).

[] J. Yu, P. Shi, J. Liu, C. Lin, Neuroadaptive finite-time control for nonlinear mimo systems with input constraint, IEEE Transactions on Cybernetics (2020).

[] C. Fu, Q.-G. Wang, J. Yu, C. Lin, Neural network-based finite-time command filtering control for switched nonlinear systems with backlash-like hysteresis, IEEE Transactions on Neural Networks and Learning Systems (2020).

[] G. Cui, J. Yu, Q.-G. Wang, Finite-time adaptive fuzzy control for mimo nonlinear systems with input saturation via improved command-filtered backstepping, IEEE Transactions on Systems, Man, and Cybernetics: Systems (2020).

[] J.-M. Bastin, G.and Coron, Stability and boundary stabilization of 1-d hyperbolic systems, volume 88, Springer, 2016.

[] L. Hong, I. Girsang, J. S. Dhupia, Identification and control of stick-slip vibrations using kalman estimator in oil-well drill strings, Journal of Petroleum Science and Engineering 140 (2016) 119-127.

[] L. W. Ledgerwood, R. Spencer, O. Matthews, J. A. Bomidi, J. Mendoza, J. Hanson, The effect of bit type on reactive torque and consequent tool-face-control anomalies, SPE Drilling \& Completion 31 (2016) 095-105.

[] I. J. Inyang, J. F. Whidborne, M. T. Bayliss, Directional drilling attitude control with input disturbances and feedback delay, IFAC-PapersOnLine 50 (2017) 1409-1414.

[] N. Kazemi, S. Nejadi, J. Auriol, J. Curkan, R. J. Shor, K. A. Innanen, S. M. Hubbard, I. D. Gates, Advanced sensing and imaging for efficient energy exploration in complex reservoirs, Energy Reports 6 (2020) 3104-3118.

[] K.-D. Chen, J.-Q. Chen, D. Hong, X.-Y. Zhong, Z.-B. Cheng, Q.-H. Lu, J.-P. Liu, Z.-H. Zhao, G.-X. Ren, Efficient and high-fidelity steering ability prediction of a slender drilling assembly, Acta Mechanica 230 (2019) 3963-3988.

[] C. Germay, N. van de Wouw, H. Nijmeijer, R. Sepulchre, Nonlinear Drillstring Dynamics Analysis, SIAM Journal on Applied Dynamical Systems 8 (2009) 527-553. URL: http://epubs . siam.org/doi/abs/10.1137/060675848. doi $10.1137 / 060675848$.

[] E. Cayeux, On the Importance of Boundary Conditions for Real-Time Transient Drill-String Mechanical Estimations, in: IADC/SPE Drilling Conference and Exhibition, Society of Petroleum Engineers, 2018. URL: http://www.onepetro.org/doi/10.2118/189642-MS doi 10.2118/189642-MS

[] U. J. F. Aarsnes, O. M. Aamo, M. Krstic, Extremum seeking for real-time optimal drilling control, in: 2019 American Control Conference (ACC), IEEE, 2019, pp. 5222-5227.

[] E. Detournay, T. Richard, M. Shepherd, Drilling response of drag bits: theory and experiment, International Journal of Rock Mechanics and Mining Sciences 45 (2008) 1347-1360.

[] E. Detournay, P. Defourny, A phenomenological model for the drilling action of drag bits, International Journal of Rock Mechanics and Mining Sciences \& Geomechanics Abstracts 29 (1992) 13-23. URL: http: //linkinghub.elsevier.com/retrieve/pii/0148906292910413 doi 10.1016/0148-9062(92)91041-3.

[] J. Auriol, U. J. F. Aarsnes, R. Shor, Self-tuning torsional drilling model for real-time applications, in: American Control Conference, 2020.

[] R. J. LeVeque, Finite volume methods for hyperbolic problems, Cambridge university press, 2002.

M. Krstic, A. Smyshlyaev, Boundary Control of PDEs: A Course on Backstepping Designs, SIAM, 2008.

[] J.-M. Coron, R. Vazquez, M. Krstic, G. Bastin, Local Exponential $H^{2}$ Stabilization of a 2 x 2 Quasilinear Hyperbolic System Using Backstepping, SIAM Journal on Control and Optimization 51 (2013) 2005-2035. URL: http://ieeexplore.ieee.org/document/6161075/http://epubs.siam.org/doi/10.1137/120875739 doi 10. 1137/120875739 arXiv:1208.6475.

[] T. Richard, F. Dagrain, E. Poyol, E. Detournay, Rock strength determination from scratch tests, Engineering Geology 147 (2012) 91-100.

[] P. Sharma, T. Singh, A correlation between p-wave velocity, impact strength index, slake durability index and uniaxial compressive strength, Bulletin of Engineering Geology and the Environment 67 (2008) 17-22.

[] J. Auriol, N. Kazemi, K. Innanen, R. J. Shor, Combining formation seismic velocities while drilling and a pdeode observer to improve the drill-string dynamics estimation, in: 2020 American Control Conference (ACC), IEEE, 2020, pp. 3120-3125. 
[] J. Rector III, B. Hardage, Radiation pattern and seismic waves generated by a working roller-cone drill bit, Geophysics 57 (1992) 1319-1333.

[] B. Goodway, T. Chen, J. Downton, Improved avo fluid detection and lithology discrimination using lamé petrophysical parameters; " $\lambda \rho ", " \mu \rho ", \&$ " $\lambda / \mu$ fluid stack", from p and s inversions, in: SEG Technical Program Expanded Abstracts 1997, Society of Exploration Geophysicists, 1997, pp. 183-186.

[] C. Nwankpa, W. Ijomah, A. Gachagan, S. Marshall, Activation functions: Comparison of trends in practice and research for deep learning, arXiv preprint arXiv:1811.03378 (2018).

[] J. Yosinski, J. Clune, Y. Bengio, H. Lipson, How transferable are features in deep neural networks?, in: Advances in neural information processing systems, 2014, pp. 3320-3328.

[] E. Tzeng, J. Hoffman, T. Darrell, K. Saenko, Simultaneous deep transfer across domains and tasks, in: Proceedings of the IEEE International Conference on Computer Vision, 2015, pp. 4068-4076.

[] N. Kazemi, Across-domains transferability of deep-red in de-noising and compressive sensing recovery of seismic data, arXiv preprint arXiv:2007.10250 (2020).

[] R. Courant, K. Friedrichs, H. Lewy, On the partial difference equations of mathematical physics, IBM journal of Research and Development 11 (1967) 215-234.

[] J. Auriol, Robust design of backstepping controllers for systems of linear hyperbolic PDEs, Ph.D. thesis, PSL Research University, 2018.

[] N. Kazemi, E. Bongajum, M. D. Sacchi, Surface-consistent sparse multichannel blind deconvolution of seismic signals, IEEE Transactions on geoscience and remote sensing 54 (2016) 3200-3207.

[] U. J. F. Aarsnes, J. Auriol, F. Di Meglio, R. J. Shor, Estimating friction factors while drilling, Journal of Petroleum Science and Engineering 179 (2019) 80-91. 